CALT-68-1832

CERN-TH.6694/92

HUTP-A061/92

USC-92/021

hepth@xxx/9211040

\title{
Extended N=2 Superconformal Structure of Gravity and W-Gravity Coupled to Matter
}

\author{
M. Bershadsky \\ Lyman Laboratory, Harvard University, Cambridge, MA 02138
}

W. Lerche

California Institute of Technology, Pasadena, CA 91125

and CERN, Geneva, Switzerland

D. Nemeschansky and N.P. Warner

Physics Department, U.S.C., University Park, Los Angeles, CA 90089

\begin{abstract}
We show that almost all string theories, including the bosonic string, the superstring and $W$-string theories, possess a twisted $N=2$ superconformal symmetry. This enables us to establish a connection between topological gravity and the field theoretical description of matter coupled to gravity. We also show how the $B R S T$ operators of the $W_{n}$-string can be obtained by hamiltonian reduction of $S L(n \mid n-1)$. The tachyonic and ground ring states of $W$-strings are described in the light of the $N=2$ superconformal structure, and the ground ring generators for the non-critical $W_{3}$-string are explicitly constructed. The relationship to $G / G$ models and quantum integrable systems is also briefly described.
\end{abstract}

CERN-TH.6694/92

October 1992 


\section{Introduction}

During the past decade our understanding of string theory and two-dimensional quantum gravity has grown considerably. Much of the recent progress was stimulated by the success of matrix models [1]. Still more recently, Witten has shown that topological field theories can be used to describe two-dimensional quantum gravity [2]. In this approach the unperturbed correlation functions of the latter are identified with the intersection numbers of cycles on the moduli space of Riemann surfaces. The matrix model and the topological field theory approach are now known to be equivalent to each other [3].

One of the more important problems in two-dimensional string theory is to reconstruct the results of topological gravity (or matrix models) directly from the continuum, field theoretic approach in which matter fields are directly coupled to the Liouville field. In the topological formulation of two-dimensional quantum gravity coupled to matter [甘], the relevant matter fields come from a twisted $N=2$ superconformal minimal model [5]. In this paper we establish a direct connection between the continuum formulation of matter models coupled to gravity and such twisted $N=2$ superconformal models. Several authors have observed (see, for example, [6]) that the BRST current and the anti-ghost field, $b(z)$, generate an algebra that is reminiscent of, but apparently not identical to, the $N=2$ superconformal algebra. It does not, however, seem to be broadly appreciated that the BRST current can be modified by total derivative terms so that the anti-ghost and the physical BRST current exactly generate a topologically twisted [5] $N=2$ superconformal algebra. Indeed, there are several ways in which this modification can be done. This observation was first made in [7]. The underlying $N=2$ superconformal symmetry is quite generic, and is present in every string theory.

Standard two-dimensional gravity is moderately well understood, and thus interest is now being focussed on other string theories for which the matrix model discription, or a clear topological description, is either deficient or completely lacking. This happens, for example, when the conformal matter system has central charge larger than one. In these circumstances the conformal matter system may have a larger symmetry, and it can often be coupled to some extended background geometry. In addition to well known generalizations to super-geometry, one can also try to define a 
" $W$-geometry", in which conformal $W$-matter is coupled to $W$-gravity. We will show that all $W$-gravity theories have a twisted $N=2$ super- $W$ conformal symmetry. In fact, this phenomenon is quite general: Gauging the local $W$-symmetry automatically leads to the existence of an $N=2$ supersymmetry. Perhaps more importantly, one can reverse this perspective, and use a manifestly $N=2$ superconformal hamiltonian reduction to obtain the the $B R S T$ structure for a general $W$-gravity coupled to $W$ matter. In section 2 of this paper we will explicitly construct the (extended) $N=2$ superconformal generators for the non-critical $W$-strings for which the $B R S T$ current is explicitly known. In section 3 we will show how the general BRST current for noncritical " $W_{n}$-strings" can be obtained by hamiltonian reduction of the affine super-Lie algebra $S L(n \mid n-1)$. This approach makes manifest, and directly exploits, the $N=2$ superconformal structure. In addition, hamiltonian reduction establishes a direct relation between $W$-gravities and topological field theories based upon Kazama-Suzuki models [8] obtained from $S U(n)_{k} / U(n-1)$.

In section 4 of this paper we will describe the relationship between the spectrum of the standard two-dimensional string and the $N=2$ superconformal minimal models. Specifically, we show that the coupling between topological matter and topological gravity amounts to effectively undoing one of the $N=2$ superconformal screening operations, thereby producing a field theory that is equivalent to standard two-dimensional gravity. We also discuss the generalization of this to $W$-strings. One can then use some of the machinery of $N=2$ superconformal field theories to obtain a new perspective on $W$-string theory, and most particularly upon ground rings [9]. (The ground ring and extra states for the non-critical $W_{3}$-string will be discussed in Appendices A and B of this paper.) There is, however, one subtlety: the complete physical spectrum of the string theory generically forms a non-unitary representation of the $N=2$ superconformal algebra. As a result, some of the standard theorems about chiral rings no longer apply.

When the $N=2$ superconformal structure of string theories is combined with the known relationship between $N=2$ superconformal models and topological $G / G$ models [10] we also see how the continuum field theoretic approach to two-dimensional quantum $W$-gravity is related to the approach based upon topological $G / G$ models [11-13]. In particular, we argue that the topological $G / G$ models and the standard continuum description of $W$-gravity simply yield string theories at different values 
of the cosmological constant. We also show that a non-critical $W$-string possesses an infinite number of conserved $W$-charges, even in the presence of a cosmological constant perturbation. These issues will be discussed in section 4 .

There are also natural questions about further generalizations of this hidden superconformal structure. One obvious thing to consider is the BRST structure of two-dimensional, $N=1$ supergravity. We discuss this in the next section and show that there is in fact a $N=3$ superconformal symmetry. We similarly expect that the $N=2$ supergravity theories [14 actually have an $N=4$ superconformal structure. It would be very interesting to see whether this supersymmetric extension of symmetry algebras has some analogue in higher dimensional field theories.

\section{The $N=2$ superconformal structure of $W$-strings}

\subsection{Notation and conventions}

We begin by introducing our notation and setting our conventions. The $W$ Liouville (or Toda) system will be realized by $n-1$ free bosons, $\phi_{L, i}, i=1,2, \ldots, n-1$, with operator product: $\phi_{L, i}(z) \phi_{L, j}(w)=-\delta_{i j} \log (z-w)$. It is natural to think of them as generating a Cartan subalgebra of $S L(n)$. The energy momentum tensor is given by

$$
T_{L}=-\frac{1}{2} \sum_{i=1}^{n-1}\left(\partial \phi_{L, i}\right)^{2}+\beta_{0}\left(\rho \cdot \partial^{2} \phi_{L}\right)
$$

where $\rho$ is the Weyl vector ${ }^{\star}$ of $S L(n), \beta_{0} \equiv \frac{(t+1)}{\sqrt{t}}$, and $t$ is a parameter.

It is convenient to parametrize the central charge of the matter system as:

$$
c^{M}=(n-1)\left[1-n(n+1) \frac{(t-1)^{2}}{t}\right] .
$$

At this point we are not making any assumptions about the structure of the matter system. It can be an arbitrary conformal field theory with an additional $W_{n}$ symmetry. At some points we will find it useful to realize the matter system in terms of free

* The Weyl vector is defined to be half the sum of the positive roots. We normalize roots to have squared-length equal to two, and so for $S L(2)$ we take $\rho=1 / \sqrt{2}$. 
bosons, $\phi_{M, i}, i=1,2, \ldots n-1$, with $\phi_{M, i}(z) \phi_{M, j}(w)=-\delta_{i j} \log (z-w)$ and with energy momentum tensor:

$$
T_{M}=-\frac{1}{2} \sum_{i=1}^{n-1}\left(\partial \phi_{M, i}\right)^{2}+i \alpha_{0}\left(\rho \cdot \partial^{2} \phi_{M}\right)
$$

where $\alpha_{0} \equiv \frac{(1-t)}{\sqrt{t}}$. Note that $W_{n}$-minimal matter models, which we will denote by $\mathcal{M}_{p, q}^{(n)}$, correspond to $t=q / p$. To get these minimal models, one must also perform a Felder BRST reduction ${ }^{\dagger}$ using the screening currents:

$$
S_{M}^{ \pm}\left(\alpha_{i}\right)=e^{-i \alpha_{ \pm} \alpha_{i} \cdot \phi_{M}}
$$

where the $\alpha_{i}$ 's are simple roots of $S L(n)$ and $\alpha_{+} \equiv \sqrt{t}, \alpha_{-} \equiv-1 / \sqrt{t}[15]$. In the free field realization, the primary fields are represented by vertex operators

$$
\Phi_{r_{i} ; s_{i}}=V_{r_{i} ; s_{i}}^{M} \equiv e^{i \alpha_{r_{i} ; s_{i}}^{M} \cdot \phi_{M}}
$$

with

$$
\alpha_{r_{i} ; s_{i}}^{M}=\sum_{j=1}^{n-1}\left[\alpha_{+}\left(r_{j}-1\right)+\alpha_{-}\left(s_{j}-1\right)\right] \lambda_{j},
$$

where $\lambda_{i}, i=1, \ldots, n-1$, are the fundamental weights. For minimal models $\mathcal{M}_{p, q}^{(n)}$, one can restrict the labels to the Kac table, that is, to $\sum r_{i} \leq p-1$ and $\sum s_{i} \leq q-1$, $s_{i} \geq 1, r_{i} \geq 1$. In addition, it will prove convenient to introduce vertex operators in the Liouville sector

$$
V_{r_{i} ; s_{i}}^{L} \equiv e^{\alpha_{r_{i} ; s_{i}}^{L} \cdot \phi_{L}}
$$

where

$$
\alpha_{r_{i} ; s_{i}}^{L}=\sum_{j=1}^{n-1}\left[-\alpha_{+}\left(r_{j}-1\right)+\alpha_{-}\left(s_{j}-1\right)\right] \lambda_{j} .
$$

For the remainder of this section there will be no need to represent the matter system by $n-1$ free bosons: it is only required that the matter system provide a $W$-algebra and have central charge given by (2.2).

$\dagger$ We will use the nomenclature "Felder $B R S T$ reduction" to distinguish such a reduction from the $B R S T$ reduction using the $B R S T$ charge of the underlying string theory. 
The ghost system consists of ghosts, $c_{j}$, and anti-ghosts, $b_{j},(j=1,2, \ldots, n-1)$ of spins $-j$ and $j+1$ respectively. The corresponding energy-momentum tensors are:

$$
T_{g h}^{j}(z) \equiv-(j+1) b_{j} \partial c_{j}-j\left(\partial b_{j}\right) c_{j}
$$

with central charge $c^{(j)}=-2\left(6 j^{2}+6 j+1\right)$. With the foregoing choices, the total ghost central charge of $-2(n-1)\left(2 n^{2}+2 n+1\right)$ is precisely offset by the central charge of the matter plus gravity systems: $c^{L}+c^{M}=2 n+12\left(\beta_{0}^{2}-\alpha_{0}^{2}\right) \rho^{2}=2(n-1)\left(2 n^{2}+2 n+1\right)$.

The " $W_{n}$-string" models [16 19] that we will consider are thus tensor products

$$
\mathcal{W}^{(n)} \equiv W_{n}^{\text {matter }} \otimes W_{n}^{\text {Liouville }} \otimes_{j=1}^{n-1}\left\{b_{j}, c_{j}\right\}
$$

where $\left\{b_{j}, c_{j}\right\}$ denotes the Hilbert space of the $j^{\text {th }}$ ghost system. Since the $B R S T$ charges for these $W_{n}$-string systems are only explicitly known for $n=2$ and $N=3$, we now restrict to these cases, but we will reconsider general values of $n$ in sections 3 and 5 .

\section{2. $N=2$ superconformal symmetry in two-dimensional gravity}

We start with non-critical $W_{2}$-strings, which are simply matter coupled to ordinary gravity. The matter system can be arbitrary, except that it must have conformal anomaly equal to $c^{M}=13-6(t+1 / t)$. The BRST current is well known [20]:

$$
\mathcal{J}_{B R S T}(z)=c_{1}(z)\left[T_{L}(z)+T_{M}(z)+\frac{1}{2} T_{g h}^{1}(z)\right]
$$

It has been noted by many authors that the BRST current $J_{B R S T}$ and the antighost field $b_{1}(z)$ generates an algebra that is very similar to $N=2$ superconformal algebra. Unfortunately this algebra is not closed. The operator product of BRST current with itself is singular and generates a new field $c \partial c$. However one can modify the BRST current by a total derivative term in such a manner that the operator product is regular [7]. Such a modification clearly does not affect the BRST charge. To be explicit, we introduce the $U(1)$ current:

$$
\begin{gathered}
J(z)=c_{1} b_{1}+\sqrt{\frac{2}{t}} \partial \phi_{L}, \\
-5-
\end{gathered}
$$


where $\phi_{L}$ is the Liouville field. Then, defining an "improved" BRST current,

$$
G^{+}(z)=\mathcal{J}_{B R S T}(z)-\sqrt{\frac{2}{t}} \partial\left(c_{1} \partial \phi_{L}\right)+\frac{1}{2}\left(1-\frac{2}{t}\right) \partial^{2} c_{1},
$$

the currents $G^{+}(z)$ and $J(z)$, along with

$$
T(z)=T_{L}(z)+T_{M}(z)+T_{g h}^{1}(z) \quad \text { and } \quad G^{-}(z)=b_{1}(z)
$$

close into a twisted $N=2$ superconformal algebra [5]:

$$
\begin{aligned}
T(z) \cdot T(w) & \sim \frac{2 T(w)}{(z-w)^{2}}+\frac{\partial T(w)}{(z-w)}, \\
T(z) \cdot G^{ \pm}(w) & \sim \frac{\frac{1}{2}(3 \mp 1) G^{ \pm}(w)}{(z-w)^{2}}+\frac{\partial G^{ \pm}(w)}{(z-w)}, \\
T(z) \cdot J(w) & \sim \frac{\frac{1}{3} c^{N=2}}{(z-w)^{3}}+\frac{J(w)}{(z-w)^{2}}+\frac{\partial J(w)}{(z-w)}, \\
J(z) \cdot J(w) & \sim \frac{\frac{1}{3} c^{N=2}}{(z-w)^{2}}, \quad J(z) \cdot G^{ \pm}(w) \sim \pm \frac{G^{ \pm}(w)}{(z-w)}, \\
G^{+}(z) \cdot G^{-}(w) & \sim \frac{\frac{1}{3} c^{N=2}}{(z-w)^{3}}+\frac{J(w)}{(z-w)^{2}}+\frac{T(w)+\partial J(w)}{(z-w)}, \\
G^{ \pm}(z) \cdot G^{ \pm}(w) & \sim 0 .
\end{aligned}
$$

The anomaly, which is proportional to the central charge of the corresponding untwisted $N=2$ superconformal theory, is:

$$
c^{N=2}=3\left(1-\frac{2}{t}\right) .
$$

This relation implies that the string with $c^{M}=1$ has $c^{N=2}=-3$, while the ordinary bosonic string (with $c^{M}=25$ ) is mapped onto a superconformal theory with $c^{N=2}=9$. An immediate observation [7] is that if the matter model is taken to be $\mathcal{M}_{p=1, q=k+2}^{(2)}$, then this central charge becomes $c^{N=2}=\frac{3 k}{(k+2)}$, and corresponds to the $N=2$ superconformal minimal models. We will discuss this point in more detail in section 4 .

One can also obtain a twisted $N=2$ superconformal algebra by replacing $t \rightarrow 1 / t$ throughout the foregoing discussion. Therefore the general matter-gravity system has at least two twisted $N=2$ superconformal algebras. 
It is interesting to note that the foregoing construction of the $N=2$ superconformal operators did not require any specific form for the matter part of the theory. The only requirement on the combined matter and gravity systems is the existence the $U(1)$ current that one uses to modify the BRST current. The matter system can be arbitrary and thus even the critical $(D=26)$ string theory possesses $N=2$ twisted supersymmetry. If one can find a realization of the matter sector that employs at least one boson, $\phi_{M}$, then one can use this, instead of $\phi_{L}$, to modify the $B R S T$ current ${ }^{\star}$. This choice is equivalent to simply performing the substitution $t \rightarrow-t$ and interchanging $\phi_{L}$ with $\phi_{M}$ throughout this section.

Having exposed this $N=2$ superconformal structure, it is natural to recast general questions about gravity coupled to matter in terms of $N=2$ superconformal theory. We will defer this until section 4.

The appearence of an extended superconformal structure appears to be quite general in systems of matter with extended symmetries, coupled to extended geometries.

\section{3. $N=3$ superconformal structure of $N=1$ supergravity}

In this section we will generalize the construction discussed above to superstrings. It was suggested by several authors that topological superstring theories may have $N=3$ twisted supersymmetry [22]. We will see below that any superstring theory has an $N=3$ twisted supersymmetry. The only requirement that we will make is that the combined super-matter and supergravity system possess a $U(1)$ supercurrent that can be used to improve the $B R S T$ current. We parametrize the matter central charge as

$$
\widehat{c}=5-2(t+1 / t)
$$

and in this parametrization the superconformal minimal models correspond to rational $t=q / p$.

The modified BRST current $J_{B R S T}$ (spin 1) and the diffeomorphism ghost $b$ (spin 2) together with $N=1$ supercurrent $G$ (spin 3/2) constitute three super-generators

$\star$ This possibility is utilized in a forthcoming paper by C.Vafa and S.Mukhi to relate the $c^{M}=1$ string to a superconformal $S L(2) / U(1)$ coset model [21]. 
of $N=3$ twisted superalgebra. As in the bosonic case, the stress-energy tensor $T$, modified BRST current $J_{B R S T}$, the ghost $b$ and the modified ghost current $H$ make an $N=2$ multiplet. The original $N=1$ supersymmetry remains unbroken so that we can use $N=1$ superformalism

$$
\begin{aligned}
\mathcal{J}_{B R S T} & =j_{B R S T}+\theta J_{B R S T}, \\
\mathcal{T} & =G+\theta T, \\
\mathcal{B} & =\beta+\theta b, \\
\mathcal{H} & =h+\theta H .
\end{aligned}
$$

The lower components of the superfield make another $N=2$ multiplet. For later convenience we also introduce the super-ghost field $\mathcal{C}=c+\theta \gamma$. The lower component of the BRST current $j_{B R S T}$, modified $U(1)$ charge and the super-diffeomorphism ghost $\beta$ become the $S L(2)$ currents of $N=3$ twisted algebra.

In order to identify $J_{B R S T}$ with the supercurrent and $j_{B R S T}$ with one of the $S L(2)$ currents, we will modify the super-BRST current by total derivative piece to ensure the operator products $G^{+}(z) G^{+}(w)$ and $K^{+}(z) K^{+}(w)$ are regular. Namely

$$
\begin{gathered}
K^{+}+\theta G^{+}=\mathcal{J}_{B R S T}+\Delta \mathcal{J}_{B R S T} \\
\mathcal{J}_{B R S T}=\left(\mathcal{T}+\frac{1}{4} D \mathcal{C} D \mathcal{B}-\frac{3}{4} \partial \mathcal{C B}\right) \mathcal{C}-\frac{3}{4} D(\mathcal{C} D \mathcal{C B}) \\
\Delta \mathcal{J}_{B R S T}=D(\mathcal{C} D \mathcal{C B})-\frac{1}{2 t} D \partial \mathcal{C}-\frac{1}{\sqrt{t}} D(\mathcal{C} \partial \Phi)+\frac{1}{2 \sqrt{t}} D(D \mathcal{C} D \Phi)
\end{gathered}
$$

where $D=\partial_{\theta}+\theta \partial_{z}$ and $\Phi=\phi+\theta \psi$ is the super-Liouville field.

We are not going to describe these rather tedious calculations in detail, but we will just present the results. The three $S L(2)$ currents are given as follows

$$
\begin{aligned}
K^{+} & =2 i\left(j_{B R S T}-\frac{1}{2 t} \partial \gamma-\frac{1}{2 \sqrt{t}} \gamma \partial \phi+\frac{1}{2} \partial c \psi+\frac{1}{\sqrt{t}} c \partial \psi\right), \\
K^{3} & =c b-\beta \gamma+\frac{1}{\sqrt{t}} \partial \phi \quad, \quad K^{-}=2 i \beta
\end{aligned}
$$

It is straightforward to check that these currents make an honest $S L(2)$ current algebra at level $-1 / t$. There are also three super-generators

$$
\begin{gathered}
G^{+}=2 i\left(J_{B R S T}-\frac{1}{2 t} \partial^{2} c-\frac{1}{\sqrt{t}} \partial(c \partial \phi)+\frac{1}{2 \sqrt{t}} \partial(\gamma \psi)+\partial(c \beta \gamma)\right), \\
G^{3}=2\left(G_{m+l}+G_{\text {ghost }}\right), \quad G^{-}=-2 i b \\
-8-
\end{gathered}
$$


and there is also an additional free fermion $F=\beta c-\frac{1}{\sqrt{t}} \psi$.

Undoing the twisting: $T \rightarrow T-\frac{1}{2} \partial K^{3}$ and $G^{3} \rightarrow G^{3}+\partial F$, one obtains the $N=3$ superconformal algebra, described by Seiberg and Schwimmer [23]. All fields become conformal primary fields. The supercurrents $G^{a}$ are almost in the adjoint representation of $S L(2)$

$$
K^{a} G^{b} \sim \frac{1}{(z-w)} f_{c}^{a b} G^{c}-F \frac{\eta^{a b}}{(z-w)^{2}},
$$

modulo the fact that there is a central term proportional to the free fermion. In the operator products between supercurrents one generates the stress-energy tensor and $S L(2)$ currents

$$
G^{a} G^{b} \sim \frac{1}{(z-w)^{2}} f_{c}^{a b} K^{c}+\frac{1}{(z-w)}\left(2 \eta^{a b} T+2 f_{c}^{a b} \partial K^{c}\right)-\frac{2 / t}{(z-w)^{3}} \eta^{a b}
$$

In the operator products between supercurrents and free fermion one generates the $S L(2)$ currents

$$
G^{a} F \sim-\frac{1}{(z-w)} K^{a}
$$

The remaining operator products are

$$
F F \sim \frac{-1 / t}{(z-w)} \text { and } K^{a} F \sim R e g
$$

The resulting $N=3$ algebra has a conformal anomaly equal to $c^{N=3}=-3 / t$. As in section 2 of this paper, one can can replace $t \rightarrow 1 / t$ in the foregoing calculation and thereby obtain a theory with $c^{N=3}=-3 t$. In the derivation above we did not make any assumption about the structure of $N=1$ matter system. That means that even ten-dimensional critical superstring theory has $N=3$ twisted supersymmetry.

The discussion above implies that superconformal matter systems coupled to supergravity are closely related to topological $N=3$ twisted models. In order to construct these models explicitly one has to know the representation theory of $N=3$ superconformal algebra. We postpone the discussion of the representation theory of $N=3$ algebra to one of our future publications.

It is a little puzzling to have an $N=3$ algebra. We know quite well that for $\sigma$-models, $N=3$ supersymmetry automatically implies $N=4$ [24]. In fact, in our case there are some reasons to believe that the $N=3$ algebra discussed above can be promoted to $N=4$. This question is also under investigation. 


\section{4. $\quad$ =2 superconformal structure of $W_{3}$-gravity}

A BRST charge for the $W_{3}$-string, $\mathcal{W}^{(3)}$, was found in [25,26]. This charge is nilpotent if the total central charge vanishes (i.e., if $c^{L}+c^{M}=-c^{g h}=100$ ), and it is given by $\mathcal{Q}_{B R S T}=\oint \frac{d z}{2 \pi i} \mathcal{J}_{B R S T}(z)$, where

$$
\begin{aligned}
\mathcal{J}_{B R S T} & =c_{2}\left[\frac{1}{b_{L}} W_{L}+\frac{i}{b_{M}} W_{M}\right]+c_{1}\left[T_{L}+T_{M}+\frac{1}{2} T_{g h}^{1}+T_{g h}^{2}\right] \\
& +\left[T_{L}-T_{M}\right] b_{1} c_{2}\left(\partial c_{2}\right)+\mu\left(\partial b_{1}\right) c_{2}\left(\partial^{2} c_{2}\right)+\nu b_{1} c_{2}\left(\partial^{3} c_{2}\right),
\end{aligned}
$$

and where $b_{L, M}^{2} \equiv \frac{16}{5 c_{L, M}+22}$ and $\mu=\frac{3}{5} \nu=\frac{1}{10 b_{L}{ }^{2}}\left(1-17 b_{L}{ }^{2}\right)$. In this equation, $T_{L, M}$ and $W_{L, M}$ denote the usual stress tensors and $W$-generators of the Liouville and matter sectors, and $T_{g h}^{i}$ are the stress tensors of the ghosts.

The BRST current, $\mathcal{J}_{B R S T}$, can be identified with the $N=2$ supercurrent, $G^{+}$, if one adds a total derivative piece to $\mathcal{J}_{B R S T}$ so as to ensure that $G^{+}(z) G^{+}(w) \sim 0$. That is, for

$$
G^{+}=\mathcal{J}_{B R S T}+\Delta \mathcal{J}
$$

we find:

$$
\begin{aligned}
\Delta \mathcal{J} & =\partial\left[-c_{1} J+2 i \sqrt{\frac{t}{3}} b_{1} c_{1} c_{2} J+i \frac{(1+t)}{2} \sqrt{\frac{3}{t}} b_{1} c_{1}\left(\partial c_{2}\right)\right. \\
& -i \frac{(3+2 t)}{\sqrt{3 t}} b_{1}\left(\partial c_{1}\right) c_{2}-\frac{\left(7 t^{2}-10 t-15\right)}{4 t} b_{1}\left(\partial^{2} c_{2}\right) c_{2}+i \frac{(t-9)}{\sqrt{3 t}} b_{2}\left(\partial c_{2}\right) c_{2} \\
& -i \frac{(3+4 t)}{\sqrt{3 t}}\left(\partial b_{1}\right) c_{1} c_{2}-\frac{3\left(4 t^{2}-2 t-3\right)}{2 t}\left(\partial b_{1}\right)\left(\partial c_{2}\right) c_{2}+\frac{(t-3)}{t}\left(\partial c_{1}\right) \\
& +i \frac{1}{2 \sqrt{3 t}} c_{2}\left[2 t J^{2}-3(t-5) T_{L}-3(t-1) T_{M}-6(1+t) \partial J\right] \\
& \left.+i \frac{(1+t)}{2} \sqrt{\frac{3}{t}}\left(\partial c_{2}\right) J-i \frac{\left(t^{2}-4 t-1\right)}{2 t} \sqrt{\frac{3}{t}}\left(\partial^{2} c_{2}\right)+t b_{1}\left(\partial c_{2}\right) c_{2} J\right] .
\end{aligned}
$$

Here, $J$ in (2.28) denotes the $U(1)$ current:

$$
J=c_{1} b_{1}+c_{2} b_{2}+\frac{3}{\sqrt{t}}\left(\lambda_{1} \cdot \partial \phi_{L}\right)+\frac{i}{2} \sqrt{\frac{3}{t}}(t-1) \partial\left[b_{1} c_{2}\right],
$$

where $\lambda_{1}$ denotes the first fundamental weight of $S U(3)$. In deriving (2.28) there is a choice of sign, and the other choice leads to the conjugate fundamental weight, $\lambda_{2}$, in (2.29). 
It is straightforward, but rather tedious, to check that $J, G^{+}, G^{-} \equiv b_{1}$, and $T \equiv T_{M}+T_{L}+T_{g h}^{1}+T_{g h}^{2}$ generate a (topologically twisted) $N=2$ superconformal algebra (2.15), with anomaly

$$
c^{N=2}=6\left(1-\frac{3}{t}\right)
$$

It is analogous to the $N=2$ superconformal algebra that we found for matter models coupled to ordinary gravity. However, since we are now coupling models with extended symmmetry to $W$-gravity, it is natural to expect an extension of the $N=2$ superconformal algebra. One may indeed check that the BRST multiplet of currents

$$
\begin{aligned}
& V_{2}=\alpha\left[(2+t)(3-t) T_{M}-\left(t^{2}+4 t-24\right) T_{L}+i \frac{3 t}{\sqrt{3 t}}(5 t-18) T_{L} b_{1} c_{2}\right. \\
&-\frac{t}{6}(t-12) J^{2}+i \frac{t^{2}(5 t-18)}{3 \sqrt{3 t}} J^{2} b_{1} c_{2}-\frac{t(5 t-18)}{3} J b_{1} c_{1}-i \frac{t(t-3)(5 t-18)}{6 \sqrt{3 t}} J b_{1}\left(\partial c_{2}\right) \\
&+i \frac{2 t^{2}(5 t-18)}{3 \sqrt{3 t}} J\left(\partial b_{1}\right) c_{2}-\frac{2 t(5 t-18)}{3} b_{1} b_{2} c_{1} c_{2}+i \frac{t(9+5 t)(5 t-18)}{3 \sqrt{3 t}} b_{1} b_{2}\left(\partial c_{2}\right) c_{2} \\
&-i \frac{t(1+t)(5 t-18)}{\sqrt{3 t}} b_{1} c_{2}(\partial J)+\frac{\left(11 t^{2}-24 t-36\right)}{3} b_{1}\left(\partial c_{1}\right)-i \frac{(5 t-18)\left(t^{2}-6 t-9\right)}{3 \sqrt{3 t}} b_{1}\left(\partial^{2} c_{2}\right) \\
&+i \frac{\sqrt{3 t}(5 t-18)}{3} b_{2} c_{1}+\frac{1}{2}\left(11 t^{2}-9 t-90\right) b_{2}\left(\partial c_{2}\right)+\frac{1}{6}\left(31 t^{2}-81 t-90\right)\left(\partial b_{1}\right) c_{1} \\
&+i \frac{t(3+7 t)(5 t-18)}{3 \sqrt{3 t}}\left(\partial b_{1}\right) b_{1} c_{1} c_{2}+\frac{1}{2}(1+t)(3 t-5)(5 t-18)\left(\partial b_{1}\right) b_{1}\left(\partial c_{2}\right) c_{2} \\
&-i \frac{1}{12 \sqrt{3 t}}(5 t-18)\left(23 t^{2}-6 t-45\right)\left(\partial b_{1}\right)\left(\partial c_{2}\right)+\frac{1}{3}\left(16 t^{2}-27 t-90\right)\left(\partial b_{2}\right) c_{2} \\
&\left.-i \frac{1}{6 \sqrt{3 t}}(5 t-18)\left(5 t^{2}-9\right)\left(\partial^{2} b_{1}\right) c_{2}+\frac{1}{6}\left(13 t^{2}-72-24 t\right)(\partial J)\right] \\
& F_{2}^{+}=-\left[\mathcal{Q}_{B R S T}, V_{2}\right] \\
& F_{3}^{-}=\left[\left(G^{-}\right)-1, V_{2}\right]=\alpha\left[-\frac{2}{3} t(3-2 t) b_{1} J+\frac{2}{3} t(5 t-18) b_{1} b_{2} c_{2}\right. \\
&\left.\quad+i \frac{t^{2}(5 t-18)}{\sqrt{3 t}}\left(\partial b_{1}\right) b_{1} c_{2}-i \frac{t(5 t-18)}{\sqrt{3 t}} b_{2}-\frac{3}{2}\left(3 t^{2}-9 t-2\right)\left(\partial b_{1}\right)\right] \\
& W_{3}=\frac{1}{2}\left\{\mathcal{Q}_{B R S T}, F_{3}^{-}\right\}
\end{aligned}
$$

forms a $W_{3}$ extension [27,28 of the (topologically twisted) $N=2$ superconformal algebra. The currents are correctly normalized, i.e., $V_{2}(z) V_{2}(w) \sim \frac{\frac{1}{2} c^{N=2}}{(z-w)^{4}}+\ldots$, if one sets $\alpha=[(t-4)(t+2)(2 t-3)(5 t-18) / 3]^{-1 / 2}$. Note that this $N=2$ super- $W_{3}$ algebra exists for generic ${ }^{\star} t$. Note also that the matter system enters only via its $W$ currents and therefore the foregoing statements apply to any $W_{3}$ matter system.

* At special values of $t$, the algebra may truncate. For instance, for $t=4$, the $V$ multiplet becomes null and the algebra truncates to the $N=2$ superconformal algebra. 
For the special minimal $W$-matter models $\mathcal{M}_{1, q}^{(3)}, t$ is integer and writing $t=$ $q=k+3$, the central charge in (2.30) becomes identical to the central charge of the $N=2$ superconformal coset models, or Kazama-Suzuki models [8], based on cosets $S U(3)_{k} / U(2)$. This is natural in view of our conjecture [25] that for general $n$, the models $\mathcal{M}_{1, q=n+k}^{(n)}$ coupled to $W_{n}$-gravity should be closely related to (or even be equivalent to) topologically twisted $N=2$ superconformal coset models based on $S U(n)_{k} / U(n-1)$, coupled to topological $W_{n}$-gravity. It is indeed well-known [29,27,28, 30 that the Kazama-Suzuki model based on $S U(n)_{k} / U(n-1)$ has an $N=2$ super- $W_{n}$ algebra as its maximal chiral algebras, and has a central charge

$$
c^{N=2}=3 \frac{(n-1) k}{n+k} .
$$

One thus suspects that, in general, $W_{n}$-matter models coupled to $W_{n}$-gravity should exhibit an $N=2$ superconformal $W_{n}$ symmetry, with central charge (2.32). That is, besides the spin one supermultiplet, or BRST multiplet, $\left(J, G^{+}, G^{-}, T\right)$, there should also be, for each spin $s$, a supermultiplet of of currents of the form

$$
\mathcal{V}_{s}=\left\{\begin{array}{c}
V_{s} \\
F_{s}^{+}=-\left[\mathcal{Q}_{B R S T}, V_{s}\right] \\
F_{s+1}^{-}=\left[\left(G^{-}\right)_{-1}, V_{s}\right] \\
W_{s+1}=\frac{1}{2}\left\{\mathcal{Q}_{B R S T}, F_{s+1}^{-}\right\}
\end{array}\right\} \quad s=2, \ldots, n-1
$$

The currents in this multiplet are composed of matter and Liouville $W$-currents, the $U(1)$ current and the ghosts. In the next section we will give an explicit construction of such "non-standard" $N=2$ super- $W_{3}$ algebras and we will also establish the presence of $N=2$ superconformal $W_{n}$ symmetry in general.

It appears that $N=2$ superconformal extensions of $W$-algebras carry some intrinsic notion of $W$-geometry. In particular, one can immediately infer the full structure of the descent equations of $W_{n}$-gravity. For example, for $W_{3}$ one can associate with each BRST invariant operator $\Phi^{(0)}$ fields at adjacent ghost numbers in the following manner (the superscripts label relative ghost number):

$$
\begin{aligned}
\Phi_{1}^{(-1)}= & \left(G^{-}\right)_{-1} \Phi^{(0)} \\
\Phi_{2}^{(-1)}= & \left(F_{3}^{-}\right)_{-2} \Phi^{(0)} \sim\left[\left(b_{2}\right)_{-2}+\ldots\right] \Phi^{(0)} \\
\Phi^{(-2)}= & \left(F_{3}^{-}\right)_{-2}\left(G^{-}\right)_{-1} \Phi^{(0)} \sim\left[\left(b_{2}\right)_{-2}\left(b_{1}\right)_{-1}+\ldots\right] \Phi^{(0)} \\
\Phi^{(-3)}= & \left(F_{3}^{-}\right)_{-1}\left(F_{3}^{-}\right)_{-2}\left(G^{-}\right)_{-1} \Phi^{(0)} \sim\left[\left(b_{2}\right)_{-1}\left(b_{2}\right)_{-2}\left(b_{1}\right)_{-1}+\ldots\right] \Phi^{(0)} \\
& \quad \ldots, \text { etc. }
\end{aligned}
$$


From the explicit form [27.28] of the $N=2 W_{3}$ algebra it is then clear that these fields satisfy

$$
\begin{aligned}
{\left[\mathcal{Q}_{B R S T}, \Phi^{(0)}\right]=} & 0 \\
\left\{\mathcal{Q}_{B R S T}, \Phi_{1}^{(-1)}\right\}= & L_{-1} \Phi^{(0)} \\
\left\{\mathcal{Q}_{B R S T}, \Phi_{2}^{(-1)}\right\}= & 2\left\{\left(W_{3}\right)_{-2}+\left(V_{2}\right)_{-2}\right\} \Phi^{(0)} \\
{\left[\mathcal{Q}_{B R S T}, \Phi^{(-2)}\right]=} & 2\left\{\left(W_{3}\right)_{(-2)}+\left(V_{2}\right)_{-2}\right\} \Phi_{1}^{(-1)}-L_{-1} \Phi_{2}^{(-1)} \\
\left\{\mathcal{Q}_{B R S T}, \Phi^{(-3)}\right\}= & \left\{2\left(W_{3}\right)_{-1}+\left(V_{2}\right)_{-1}\right\} \Phi^{(-2)}-\left(F_{3}^{-}\right)_{-1}\left[\mathcal{Q}_{B R S T}, \Phi^{(-2)}\right] \\
& \ldots, \text { etc. }
\end{aligned}
$$

Such descent equations generalize those of topological gravity [2, 31], and will be important for defining integrals over $W$-moduli space [25] in correlation functions.

\section{Hamiltonian reduction}

In the last section we presented several examples of twisted $N=2$ superconformal algebras. Somewhat surprisingly, the free field realization of the $N=2$ superconformal algebra was not of the standard form [32,29,27]. For example, for the minimal models we found a free field realization that contained two free bosons and two fermionic fields, but the latter had conformal dimension $\frac{3}{2}$ and $-\frac{1}{2}$. In this section we show how different free field realizations of the $N=2$ superconformal algebra, and its $W$ extensions, can be obtained by making different choices of the Borel subalgebra in a hamiltonian reduction of the superalgebra $S L(n \mid n-1)$. We present only the classical arguments, but from our analysis it is clear how one can generalize to the quantum case. From our discussion it will also be clear that two-dimensional matter coupled to $W$-gravity is naturally embedded in a constrained Wess-Zumino-Witten model based on $S L(n \mid n-1)$.

It is well known that the hamiltonian reduction of the super-algebra $S L(n \mid n-1)$ gives rise to the $W_{n}$-extension of the $N=2$ superconformal algebra [29,27]. To carry out the reduction it is convenient to use the Gauss decomposition $\mathcal{N}_{-} \oplus \mathcal{H} \oplus \mathcal{N}_{+}$of the algebra. In this decomposition one imposes constraints on the currents in $\mathcal{N}_{+}$, and the remaining degrees of freedom $\left(\mathcal{N}_{-}\right)$are gauged away. It is not surprising that different choices of Gauss decomposition can give rise to different representations of 
the same chiral algebra. The free field realization of the chiral algebra can be deduced from the Wakimoto realization of the original current algebra. Each choice of Borel subalgebra will give rise to a different Wakimoto representation of the $S L(n \mid n-1)$ current algebra and hence to a different representation of the same extended $N=2$ superconformal algebra. Below we compare two different schemes of reducing the superalgebra $S L(n \mid n-1)$. There does not exist a simple way of going from one realization to the other one. The two different free field realizations are related to each other by some highly non-trivial Bogolubov transformation.

To illustrate the basic idea of different reductions, we start by considering the simple example of $S L(2 \mid 1)$. The super-algebra $S L(2 \mid 1)$ can be realized in terms of $3 \times 3$ matrices with the following assignments of statistics

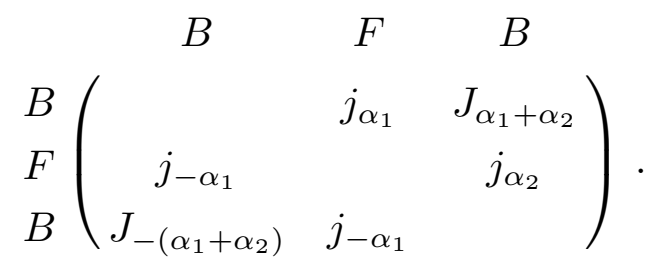

The nilpotent subalgebra $\mathcal{N}_{+}$consists of the upper triangular matrices and is generated by two fermionic currents $j_{\alpha_{1}}, j_{\alpha_{2}}$

$$
\left\{j_{\alpha_{1}}, j_{\alpha_{2}}\right\}=J_{\alpha_{1}+\alpha_{2}}
$$

The $N=2$ superconformal algebra is obtained by constraining the $S L(2 \mid 1)$ currents in the Borel sub-algebra

$$
j_{\alpha_{1}}=\chi^{\dagger}, \quad j_{\alpha_{2}}=\chi \text { and } J_{\alpha_{1}+\alpha_{2}}=1
$$

In order to make the constraints (3.3) first class we have introduced a pair of conjugate fields $\chi(z)$ and $\chi^{\dagger}(z)$ of conformal dimension $\frac{1}{2}$, with operator product:

$$
\chi(z) \chi^{\dagger}(w)=\frac{1}{z-w}
$$

We will not discuss in detail how the reduction works for this case, but we refer the reader to the original papers on hamiltonian reduction [33]. The free field realization can be obtained from a generalized Miura transformation. Consider the operator $L=\partial_{z}-\mathcal{J}(z)$, where $\mathcal{J}(z)$ is a constraint current. This operator naturally appears 
in the context of constrained Wess-Zumino-Witten models. The constrained WessZumino-Witten model is invariant under gauge transformations generated by lower triangular matrices. There are two natural choices for the gauge slice. First choose the current $\mathcal{J}$ in such a way that it is consistent with the constraints $(3.3)$

$$
\mathcal{J}(z)=\left(\begin{array}{ccc}
\frac{i}{\sqrt{2}}\left(\partial \phi_{1}+\partial \phi_{2}\right) & \chi & 1 \\
0 & i \sqrt{2} \partial \phi_{2} & \chi^{\dagger} \\
0 & 0 & \frac{i}{\sqrt{2}}\left(-\partial \phi_{1}+\partial \phi_{2}\right)
\end{array}\right)
$$

The second choice corresponds to writing $\mathcal{J}(z)$ in terms of the fields in the chiral algebra. If we perform a gauge transformation by lower triangular matrices we can reduce $(3.5)$ to the following form

$$
\mathcal{J}(z)=\left(\begin{array}{ccc}
J & 0 & 1 \\
G^{-} & 2 J & 0 \\
T-J^{2} & G^{+} & J
\end{array}\right) .
$$

It is easy to see that fields $J, G^{ \pm}$and $T$ form an $N=2$ superconformal algebra. Simple calculations lead to the following well known bosonisation rules [32] for the $N=2$ superconformal algebra:

$$
\begin{aligned}
J & =-\frac{1}{2} \chi^{\dagger} \chi+\frac{i}{\sqrt{2}} \partial \phi_{2} \\
G^{-} & =\frac{i}{\sqrt{2}} \chi^{\dagger}\left(\partial \phi_{2}-\partial \phi_{1}\right)-\partial \chi^{\dagger} \\
G^{+} & =\frac{i}{\sqrt{2}} \chi\left(\partial \phi_{2}+\partial \phi_{1}\right)+\partial \chi \\
T & =-\frac{1}{2}\left(\partial \phi_{1}\right)^{2}-\frac{1}{2}\left(\partial \phi_{2}\right)^{2}-\frac{1}{2} \chi^{\dagger} \partial \chi-\frac{1}{2} \chi \partial \chi^{\dagger}+i \sqrt{2} \partial^{2} \phi_{1}
\end{aligned}
$$

The screening currents can also be obtained from the hamiltonian reduction. In the free field realization of the superalgebra $S L(2 \mid 1)$ we have a screening operator for every simple root generator of Borel sub-algebra. After one imposes the constraints, these screening currents turn into screening currents of the $N=2$ superconformal algebra

$$
S_{\alpha_{1}}=\chi e^{i \alpha_{-}\left(\phi_{1}+i \phi_{2}\right)} \quad \text { and } \quad S_{\alpha_{2}}=\chi^{\dagger} e^{i \alpha_{-}\left(\phi_{1}-i \phi_{2}\right)}
$$

The representation of $N=2$ superconformal algebra encountered in the last section (i.e., that given in in (2.12)-(2.14)) is obtained by choosing a different Gauss 
decomposition. As before we realize $S L(2 \mid 1)$ in terms of $3 \times 3$ matrices but with the following assigments of statistics:

$$
\begin{gathered}
B \\
B \\
F
\end{gathered}\left(\begin{array}{ccc}
B & F \\
& J_{\alpha_{1}+\alpha_{2}} & j_{\alpha_{1}} \\
J_{-\left(\alpha_{1}+\alpha_{2}\right)} & & j_{-\alpha_{2}} \\
j_{-\alpha_{1}} & j_{\alpha_{2}} &
\end{array}\right)
$$

Here we have chosen our matrix in such away that a the first two rows and columns are bosonic. The Borel subalgebra is given by upper triangular matrices. These different choices of the Borel subalgebra are related to each other by a Weyl reflection with respect to a fermionic root. In this realization one of the simple roots is bosonic and the other one is fermionic. The nilpotent subalgebra $\widetilde{\mathcal{N}}_{+}$is generated by $J_{\alpha_{1}+\alpha_{2}}$ and $j_{-\alpha_{2}}$

$$
\left[J_{\alpha_{1}+\alpha_{2}}, j_{-\alpha_{2}}\right]=j_{\alpha_{1}} .
$$

General arguments imply that different reduction schemes with respect to $\mathcal{N}_{+}$generated by $j_{\alpha_{1}}, j_{\alpha_{2}}$ and $J_{\alpha_{1}+\alpha_{2}}$ (see $\left.(3.2)\right)$ and $\widetilde{\mathcal{N}}_{+}$give rise to the same $N=2$ superconformal theories [34]. As before we impose constraints on the currents in the nilpotent subalgebra $\widetilde{\mathcal{N}}_{+}$

$$
j_{\alpha_{1}}=0, \quad j_{-\alpha_{2}}=\lambda^{\dagger} \text { and } J_{\alpha_{1}+\alpha_{2}}=1,
$$

where $\lambda^{\dagger}$ is an auxiliary field with conformal dimension $\frac{3}{2}$. Surprisingly the field conjugate to $\lambda^{\dagger}$ does not appear in the constraints. To identify the conjugate field, consider how $\mathcal{J}(z)$ transforms under a gauge transformation. As before the theory is gauge invariant under $\mathcal{J}(z) \rightarrow \widetilde{\mathcal{J}}(z)=S \partial_{z} S^{-1}-S J(z) S^{-1}$, where $S$ is lower triangular matrix. The field conjugate to $\lambda^{\dagger}$ can be identified with one of the entries in matrix $S$

$$
S=\left(\begin{array}{lll}
1 & 0 & 0 \\
a & 1 & 0 \\
b & \lambda & 1
\end{array}\right) .
$$

This is nothing other than an additional gauge fixing condition that naturally appears in the context of constrained Wess-Zumino-Witten models. As before, the bosonisation rules are obtained by comparing two different gauge slices. From the constraints (3.11) we have

$$
\mathcal{J}(z)=\left(\begin{array}{ccc}
\frac{i}{\sqrt{2}}\left(\partial \phi_{1}+\partial \phi_{2}\right) & 1 & 0 \\
0 & \frac{i}{\sqrt{2}}\left(-\partial \phi_{1}+\partial \phi_{2}\right) & \lambda^{\dagger} \\
0 & 0 & i \sqrt{2} \partial \phi_{2}
\end{array}\right) .
$$


By choosing an appropriate gauge transformation one can always reduce $\widetilde{\mathcal{J}}(z)$ to the following form

$$
\widetilde{\mathcal{J}}(z)=\left(\begin{array}{ccc}
-J & 1 & 0 \\
T-J^{2} & -J & G^{-} \\
G^{+} & 0 & -2 J
\end{array}\right)
$$

One can easily convience oneself that fields $J, G^{ \pm}$and $T$ generate an $N=2$ superconformal algebra. The bosonisation rules read ${ }^{\star}$

$$
\begin{aligned}
J & =\frac{1}{2} \lambda \lambda^{\dagger}-\frac{i}{\sqrt{2}} \partial \phi_{2} \\
G^{-} & =\lambda^{\dagger} \\
G^{+} & =\frac{1}{2} \lambda \lambda^{\dagger} \partial \lambda-\frac{\lambda}{2}\left(\left(\partial \phi_{1}\right)^{2}+\left(\partial \phi_{2}\right)^{2}\right) \\
T(z) & =-\frac{1}{2}\left(\partial \phi_{1}\right)^{2}-\frac{1}{2}\left(\partial \phi_{2}\right)^{2}-\frac{3}{2} \lambda^{\dagger} \partial \lambda+\frac{1}{2} \lambda \partial \lambda^{\dagger}-\frac{i}{\sqrt{2}} \partial^{2} \phi_{1} .
\end{aligned}
$$

These classical formulas can be easily generalized to the quantum case. hamiltonian reduction also allows us to construct the screening currents. Reducing the screening currents of $S L(2 \mid 1)$ we obtain

$$
S_{\alpha_{1}+\alpha_{2}}=e^{2 i \alpha_{-} \phi_{1}} \quad \text { and } \quad S_{-\alpha_{2}}=\lambda^{\dagger} e^{-i \alpha_{-}\left(\phi_{1}+i \phi_{2}\right)}
$$

Comparing with (2.12)-(2.14) we can immediately identify $\lambda$ with the diffeomorphism ghost $c$ and $\lambda^{\dagger}$ with the anti-ghost $b$. In addition, $\phi_{1}$ can be identified with the matter free field, $\phi_{M}$, and $\phi_{2}$ can be identified with the Liouville field, $\phi_{L}$. Note that in order to make these identifications, one also has to twist the stress-energy tensor. In passing from the classical result to the quantum version, one finds that the improvement terms of (2.13) appear as quantum corrections to (3.15).

From the analysis above, it is clear how to generalize this construction to the superalgebra $S L(n \mid n-1)$. The standard representation of the super- $W_{n}$ algebra is obtained by choosing a Borel sub-algebra generated by the fermionic simple roots $\alpha_{1}, \ldots, \alpha_{2 n-2}$ of $S L(n \mid n-1)$. We can represent $S L(n \mid n-1)$ in terms of $(2 n-1) \times$ $(2 n-1)$ matrices and assign the statistics to the entries in the chess order as in (3.1),

^ These bosonisation rules have also been obtained by Lev Rozansky [35]. 
with alternating bosonic and fermionic labels. It is known that if one imposes the following constraints:

$$
\begin{aligned}
j_{\alpha_{1}}^{+} & =\chi_{1}, \quad j_{\alpha_{2}}^{+}=\chi_{2}+\chi_{1}^{\dagger}, \ldots j_{\alpha_{2 n-2}}^{+}=\chi_{2 n-3}^{\dagger} \\
J_{\alpha_{1}+\alpha_{2}}^{+} & =1, \quad J_{\alpha_{2}+\alpha_{3}}^{+}=1 \quad \ldots \quad J_{\alpha_{2 n-3}+\alpha_{2 n-2}}^{+}=1
\end{aligned}
$$

and sets the rest of the currents in the upper triangular subalgebra to zero, then the reduced theory has a super- $W_{n}$ algebra [29,27]. The whole discussion is completely parallel to that for $S L(2 \mid 1)$, and we will not describe it here.

The other representation of super- $W_{n}$ algebra utilizes a different Gauss decomposition of the superalgebra $S L(n \mid n-1)$. Again we realize $S L(n \mid n-1)$ in terms $(2 n-1) \times(2 n-1)$ matrices and but assign statistics in such a way that the first $n$ rows and $n$ columns are bosonic. The nilpotent subalgebra is generated by $2 n-3$ bosonic simple roots and one fermionic simple root. The constraints are quite complicated for the general case. First, we consider $S L(3 \mid 2)$, whose constrained form is:

$$
\begin{aligned}
& \begin{array}{lllll}
B & B & B & F & F
\end{array}
\end{aligned}
$$

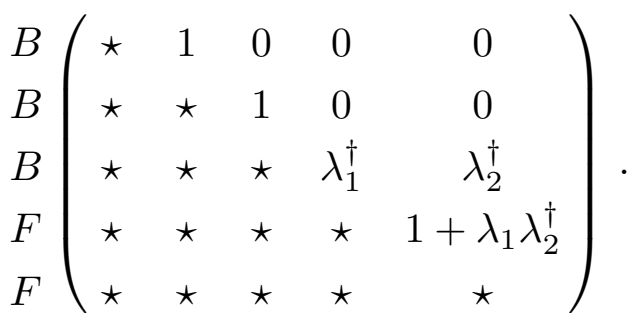

We have introduced two sets of auxiliary fields $\left(\lambda_{1}, \lambda_{1}^{\dagger}\right)$ with conformal dimension $\left(-\frac{3}{2}, \frac{5}{2}\right)$ and $\left(\lambda_{2}, \lambda_{2}^{\dagger}\right)$ with conformal dimension $\left(-\frac{1}{2}, \frac{3}{2}\right)$. As before the field, $\lambda_{2}$, that is conjugate to $\lambda_{2}^{\dagger}$ does not appear in the constraints. The field $\lambda_{2}$ has to identified with one of the parameters of the gauge transformation. To get the free field realization of super- $W_{3}$ we have to write the relation between different gauge slices. The first gauge slice is obtained by constraining all the currents from $\mathcal{N}_{-}$to zero (as in (3.13)). The second gauge slice is given by:

$$
\begin{gathered}
\left(\begin{array}{ccccc}
-J & 1 & 0 & 0 & 0 \\
0 & -J & 1 & 0 & 0 \\
\widetilde{W} & \widetilde{T} & -J & \widetilde{F}^{-} & G^{-} \\
0 & 0 & 0 & -\frac{3}{2} J & 1 \\
\widetilde{F}^{+} & G^{+} & 0 & \widetilde{S} & -\frac{3}{2} J
\end{array}\right), \\
-18-
\end{gathered}
$$


where $\widetilde{W}, \widetilde{T}, \widetilde{S}$ and $\widetilde{F}^{ \pm}$denote the currents $W, T, S$ and $F^{ \pm}$up to mixings with total derivatives and polynomials of currents of lower spin. Comparing the two gauge choices we immediately see that $G^{-}=\lambda_{2}^{\dagger}$ and $F^{-}=\lambda_{1}^{\dagger}+\ldots$. This will give rise to the classical version of the super- $W_{3}$ algebra. This algebra, is precisely the $N=2$ super$W_{3}$ algebra that was obtained in the last section. Since we have already presented the quantum version of this algebra we will not write down the classical version.

The screening currents are obtained from the screening currents of the $S L(3 \mid 2)$ current algebra via hamiltonian reduction. Since the Borel sub-algebra is generated by three bosonic roots and one fermionic root we have the following screening operators:

$$
\begin{aligned}
S_{\alpha_{1}+\alpha_{2}} & =e^{2 i \alpha_{-} \phi_{1}}, \quad S_{\alpha_{3}+\alpha_{4}}=e^{2 i \alpha_{-} \phi_{2}} \\
S_{-\left(\alpha_{2}+\alpha_{3}+\alpha_{4}\right)} & =\left(\lambda_{2}^{\dagger}+\ldots\right) e^{-i \alpha_{-}\left(\phi_{2}+i \phi_{3}\right)} \quad \text { and } \quad S_{\alpha_{2}+\alpha_{3}}=e^{2 i \alpha_{-} \phi_{4}} .
\end{aligned}
$$

It is now clear how one can generalize this construction. The constraints are nontrivial for the currents in the lower-right $n \times n$ corner. These non-trivial constraints coincide with the free field realization of the nilpotent subalgebra of a $S L(1 \mid n-1)$ submatrix. The free field realization contains $(n-1)$ pairs fermionic fields with conformal dimension $\left(-n+\frac{3}{2}, n-\frac{1}{2}\right), \ldots,\left(-\frac{1}{2}, \frac{3}{2}\right)$. As before the field with conformal dimension $-n+\frac{3}{2}$ does not appear in the constraints, but will appear as a parameter in the gauge transformation. Following our previous analysis it is not hard to see that the reduction gives rise to free field realization of super $W_{n}$-algebra where the fermionic currents $F_{i}^{-},(i=1,2, \ldots(n-1))$ are realized linearly in terms of free fermionic fields, modulo some quantum deformations. Unfortunately, the explicit form of the currents in the chiral algebra is complicated and not very illuminating. However, this construction of the super- $W$ algebra serves as a proof of the existence of BRST currents for any $W_{n}$-matter system coupled to the corresponding $W$-gravity.

\section{Chiral rings and physical states}

It is well-known that the physical states of the matter-gravity system are given by the non-trival cohomology of the operator $\mathcal{Q}_{B R S T}$. We have seen that the improved $B R S T$ current is precisely one of the supercharges, $G^{+}(z)$, of a topologically twisted $N=2$ superconformal algebra. Thus one expects that the computation of physical 
states to be very similar to the computation of the chiral ring, $\mathcal{R}$, in $N=2$ superconformal theories. However, one should note that there are several different definitions of the chiral ring, which are only equivalent for unitary theories [36]:

(i) The original definition, which was to require a field $\psi \in \mathcal{R}$ be both chiral $\left(G_{-1 / 2}^{+} \psi=0\right)$ and primary.

(ii) The cohomology of the operator $G_{-1 / 2}^{+}$.

(iii) The fields $\psi$ that obey $h_{\psi}=\frac{1}{2} q_{\psi}$, where $h_{\psi}$ and $q_{\psi}$ are the untwisted conformal weight and $U(1)$ charge. Equivalently, the conformal weight vanishes in the topologically twisted theory.

We will adopt $(i i)$ as our general definition of the "chiral ring" *. As will be seen below, chiral primaries may form only a subset of $\mathcal{R}$.

With this definition, all physical states of the non-critical $W$-string system, including discrete states 3739, are elements of $\mathcal{R}$. We will however concentrate on the ground ring and the tachyons, as only these fields are present in all the ordinary gravity theories with $c^{M} \leq 1$.

\subsection{Ordinary gravity coupled to $c \leq 1$ matter}

We start with the ground ring [9], which consists of the physical operators with vanishing ghost number. This ring is generated by ${ }^{\dagger}$

$$
\begin{aligned}
x & =\left[b c-\frac{t}{\sqrt{2 t}}\left(\partial \phi_{L}-i \partial \phi_{M}\right)\right] V_{1,2}^{L} V_{1,2}^{M}, & V_{1,2}^{L} V_{1,2}^{M} & \equiv e^{-\frac{1}{\sqrt{2 t}}\left(\phi_{L}+i \phi_{M}\right)} \\
\gamma^{0} & =\left[b c-\frac{1}{\sqrt{2 t}}\left(\partial \phi_{L}+i \partial \phi_{M}\right)\right] V_{2,1}^{L} V_{2,1}^{M}, & V_{2,1}^{L} V_{2,1}^{M} & \equiv e^{-\frac{t}{\sqrt{2 t}}\left(\phi_{L}-i \phi_{M}\right)} .
\end{aligned}
$$

* The ring multiplication may not be as simple as in the unitary case.

$\dagger$ For reasons that will soon become clear, we use the non-standard notation $x$ and $\gamma^{0}$ for the ground ring generators. 
One can represent the ring generators equivalently by replacing the matter vertex operators by their duals so that (essentially) the holomorphic and antiholomorphic combinations of the bosons are exchanged:

$$
\begin{aligned}
V_{1,2}^{L} V_{-1,-2}^{M} & \equiv e^{-\frac{1}{\sqrt{2 t}}\left(\phi_{L}-i \phi_{M}\right)} e^{i \frac{2}{\sqrt{2 t}}(1-t) \phi_{M}} \\
V_{2,1}^{L} V_{-2,-1}^{M} & \equiv e^{-\frac{t}{\sqrt{2 t}}\left(\phi_{L}+i \phi_{M}\right)} e^{i \frac{2}{\sqrt{2 t}}(1-t) \phi_{M}} .
\end{aligned}
$$

We will denote the corresponding dual representatives of the ring generators by $\widehat{x}$ and $\widehat{\gamma}^{0}$, respectively.

The generator $x$ is a primary field with respect to the $N=2$ superconformal algebra and has $U(1)$ charge $q_{x}=\frac{1}{t}$. The generator $\gamma^{0}$, on the other hand, has charge $q_{\gamma^{0}}=1$ and is, in general, not primary with respect to the $N=2$ superconformal algebra . Indeed we find:

$$
\gamma^{0}=-\left(J_{-1}+\frac{1}{t} L_{-1}\right) \gamma, \quad \gamma \equiv e^{-\frac{t}{\sqrt{2 t}}\left(\phi_{L}-i \phi_{M}\right)}
$$

where $J(z)$ is given in (2.12). (It is amusing to note that for $t=1$, which corresponds to $c^{M}=1$ matter coupled to gravity, $\gamma^{0}$ is, in fact, also primary.) This means that the ring of chiral primaries, which is given by powers of $x$, is a subset of $\mathcal{R}$, since $\mathcal{R}$ also includes powers of $\gamma^{0}$.

We saw in section 3 how the $B R S T$ operator that describes the coupling of matter to gravity can be constructed by hamiltonian reduction. The models obtained by hamiltonian reduction correspond to particular reduced $N=2$ superconformal models whose free field spectrum is reduced by a Felder BRST complex. Thus, if we wish to make a connection between the matter-gravity models and these reduced $N=2$ superconformal models, we certainly must take this Felder BRST reduction into account. In the reduced superconformal models, there are two bosonic screening operators of the form (3.16) that are easily seen to correspond precisely to the screeners (2.4) of the matter model. More interesting is the additional fermionic screener (3.16), which gives rise to the extra Felder BRST operator

$$
\begin{aligned}
\widetilde{\mathcal{Q}}= & \oint \frac{d z}{2 \pi i} b(z) \gamma(z) \\
& -21-
\end{aligned}
$$


with $\left\{\widetilde{\mathcal{Q}}, \mathcal{Q}_{B R S T}\right\}=0$. It is elementary to see that

$$
\gamma^{0}(z)=\{\widetilde{\mathcal{Q}}, \Gamma(z)\}
$$

where $\Gamma(z)$ is the (string) $B R S T$ invariant primary field

$$
\Gamma(z)=-\frac{(t+1)}{t}(\partial c)(z)-\frac{1}{\sqrt{2 t}}\left(c \partial \phi_{L}\right)(z)
$$

The complete Felder $B R S T$ operator of the $N=2$ superconformal model is the sum of $\widetilde{Q}$ and a pure matter screening operator. Since the operator $\Gamma$ has no matter components it is annihilated by the matter screening operator. Therefore the observation that $\gamma^{0}$ is $\widetilde{Q}$ exact implies that it is exact with respect to the complete Felder $B R S T$ operator of the $N=2$ superconformal theory. This means that the ground ring generator $\gamma^{0}$ is not a physical operator of the reduced $N=2$ superconformal models.

Naively one might also conclude that the ground ring generator, $x$, decouples from the $N=2$ superconformal model. Indeed one also can represent $x$ as $\widetilde{Q}$ exact

$$
x(z)=\{\widetilde{\mathcal{Q}}, F(z)\} \quad, \quad F(z)=c e^{\frac{(t+1)}{\sqrt{2 t}}\left(\phi_{L}-i \phi_{M}\right)-\frac{2}{\sqrt{2 t}} \phi_{L}}
$$

However, this does not mean that it is exact with respect to the complete Felder $B R S T$ operator. The matter part of the complete operator acts non-trivially upon $F$, and so the only conclusion one obtains from (4.7) is that $x$ is equivalent to something else modulo Felder BRST .

We thus see that one has two basic options for defining physical models: One can construct the reduced $N=2$ superconformal models, in which one employs $\widetilde{\mathcal{Q}}$ as an additional component of the Felder BRST operator, and where the only physical ground ring generator is $x$. Alternatively, one does not employ $\widetilde{\mathcal{Q}}$, in which case one obtains matter coupled to gravity with an additional ground ring generator $\gamma^{0}$.

The foregoing is parallel to the zero-momentum dilaton in the bosonic string. The dilaton takes the form $\left\{\mathcal{Q}_{B R S T},\left(c_{0}-\bar{c}_{0}\right)\right\}|0\rangle$, but one does not consider it as $B R S T$ exact since one makes the physically motivated requirement of equivariance with respect to $\left(b_{0}-\bar{b}_{0}\right)$. That is, one only allows states in the Hilbert space that are killed by $\left(b_{0}-\bar{b}_{0}\right)$. In particular, $\left(c_{0}-\bar{c}_{0}\right)|0\rangle$ is disallowed, which means that the dilaton is not the $B R S T$ variation of a physical state [40,31. 
In the present context, the point is that the requirement of not using $\widetilde{\mathcal{Q}}$ as an additional $B R S T$ operator is equivalent to the requirement of equivariance with respect to $b_{0}$. Indeed, in both formulations one does not consider $\left\{\widetilde{\mathcal{Q}}, c_{0} X\right\}$ as an exact state. Note in particular that $\Gamma(z)$ in $(4.6)$ contains $c_{0}$ explicitly, which means that $\gamma^{0}(z)$ is not $B R S T$ exact. Note also that if one imposes the condition that $b_{0}$ annihilates all physical states, the physical states must have vanishing conformal weight, since $L_{0}=\left\{\mathcal{Q}_{B R S T}, b_{0}\right\}$. Thus if one makes the additional requirement of equivariance, definition (ii) of the chiral ring implies that $(i i i)$ is also satisfied.

We now briefly turn to the tachyons, which are the physical operators with ghost number equal to one. States with non-zero ghost number fall into modules of the ground ring [9, 41]. In particular, the tachyons can be written as ground ring elements acting on certain ghost number one vacuum states $\mathcal{P}$. One must remember, however, that in free field formulations, particular representations of physical operators act only on particular spaces in a well-defined way. For the case at hand, this means that the ground ring generators can be taken to act as

$$
T_{s+t r} \sim(\widehat{x})^{s}\left(\gamma^{0}\right)^{r} \mathcal{P}
$$

where " " means equality up to appropriate screenings and up to $B R S T$ exact terms. (Equivalently, the dual representatives, $x^{s}\left(\widehat{\gamma}^{0}\right)^{r}$, can be taken to act on a dual vacuum state.) In (4.8), $\mathcal{P}$ is chosen so that all physical tachyons can be generated in this way. For minimal models $\mathcal{M}_{p, q}$, this requires taking:

$$
\mathcal{P}=c e^{\frac{1}{\sqrt{2 p q}}(q-p-1)\left(\phi_{L}-i \phi_{M}\right)} e^{\sqrt{\frac{2 p}{q}} \phi_{L}}
$$

which corresponds to the dressed matter operator with the lowest dimension. Note also that the Liouville momentum of $\mathcal{P}$ just saturates Seiberg's bound [42] of allowed Liouville momenta,

$$
\alpha^{L}<\frac{1}{\sqrt{2}} \beta_{0} \equiv \frac{t+1}{\sqrt{2 t}},
$$

so that the ground state operators indeed serve as natural vacuum states in the ghost number one sector.

For general $t$, it seems that the $N=2$ superconformal structure does not provide much additional insight in the matter models coupled to gravity. However, for the 
special matter models $\mathcal{M}_{1, q}$, coupled to gravity, there exists a direct connection with the $N=2$ minimal models. In section 2 we noted that for $t=q=k+2, k=0,1,2 \ldots$, the anomaly of the twisted $N=2$ algebra (2.15) becomes equal to the central charge of the unitary $N=2$ minimal models, $c^{N=2}=\frac{3 k}{k+2}$. This means, for integer $t \geq 2$, the reduced models are identical to the topologically twisted $N=2$ minimal models, and one can immediately infer various properties such as the structure of the chiral ring. (For general $t=q / p$, on the other hand, the level $k$ becomes fractional, which corresponds to non-minimal, non-unitary $N=2$ superconformal models. Not much can be said about such theories without further investigation.) Without performing any computation, we know that the chiral ring is characterized by the vanishing relation

$$
x^{k+1}=0
$$

The operators $x^{j}, j=0, \ldots, q-2$, are precisely the (dressed) primary fields of the minimal $\mathcal{M}_{1, q=k+2}$ models. However, the spectrum of primary fields is not uniquely defined for $\mathcal{M}_{1, q=k+2}$ models and there are reasons for including operators outside the Kac table [43,44]. These operators naturally appear in two-dimensional gravity. In order to introduce such operators, one needs to consider the equivariant cohomology. Then $\gamma^{0}$ becomes a physical operator, and it appears that (4.11) remains true [44]. Hence the chiral ring is extended to:

$$
\mathcal{R}=\left\{(x)^{j}\left(\gamma^{0}\right)^{n} \mid 0 \leq j \leq k \equiv t-2, n \geq 0\right\}
$$

This spectrum of physical operators is the same as that of topological minimal models coupled to topological gravity [4], and it was indeed long suspected that these theories should be equivalent to the $\mathcal{M}_{1, q}$ models coupled to gravity. In this context, the powers of $\gamma^{0}$ should be interpreted as gravitational descendants, so that (4.12) might be called a gravitationally extended chiral ring of the matter model.

Indeed, for $t=2$ or $k=0$, the above model turns precisely into Distler's formulation of pure topological gravity 445, where $\gamma$ in (4.3) plays the role of a commuting ghost with spin -1 . Moreover, the operators $\gamma^{0}=\partial \gamma+\ldots$ and $\Gamma$ of (4.5), (4.6) correspond to the operators denoted by $\gamma_{0}$ and $c_{0}$ in the formulation of topological gravity of ref. [31]. It is known that in topological gravity [2, 31, 46], $\gamma^{0}$ is trivial in ordinary cohomology and hence the theory is empty. However, a non-trivial theory is obtained when one employs equivariant cohomology. 
For the $\mathcal{M}_{1, q}$ models coupled to gravity, $\mathcal{P}$ can thus be interpreted as the puncture operator of topological gravity [2,31]

$$
\mathcal{P} \equiv c V_{0,1}^{L} V_{0,-1}^{M}=c \delta(\gamma),
$$

and the extra $B R S T$ operator $\widetilde{\mathcal{Q}}=\oint b \gamma$ can be interpreted as a supersymmetry charge [45].31]. Indeed, the (unperturbed) three-tachyon correlation function on the sphere can be written in the form

$$
\left\langle\mathcal{P} \mathcal{P}\left(\mathcal{P} x^{k}\right)\right\rangle=1,
$$

and this is precisely the same as the correlation function that one considers in topological gravity coupled to level $k$ topological minimal matter [4].

An important point is that for the $\mathcal{M}_{1, q}$ models, the puncture operator $\mathcal{P}$ has vanishing $N=2 U(1)$ charge, as well as vanishing conformal dimension. This means that from the $N=2$ point of view, the puncture operator is on equal footing with the identity operator of the ground ring, $\mathbf{1}$. In other words, $\mathcal{P}$ and $\mathbf{1}$ are just different copies of the vacuum. It is well-known that in free field realizations, physical operators typically appear in $M$ different copies, where $M$ is the order of the relevant Weyl group. Usually such different copies of the vacuum are given by simple vertex operators, and this is also true in the non-standard realization of the $N=2$ superconformal algebra that we use, where one copy of the vacuum just happens to be a tachyon with non-zero ghost number.

The discussion above implies that tachyons $(\widehat{x})^{s}\left(\gamma^{0}\right)^{r} \mathcal{P}$ and ground ring elements $(\widehat{x})^{s}\left(\gamma^{0}\right)^{r}$ have the same properties with respect to the $N=2$ superconformal algebra, and thus they should probably be interpreted as different realizations of the same chiral ring elements. In the $\mathcal{M}_{1, q}$ models coupled to gravity, the tachyons and the ground ring elements constitute all the physical operators ${ }^{\star}$ and therefore the ground ring describes the full set of physical operators.

Another consequence of the realization of the $N=2$ superconformal algebra in the matter-gravity system is that it suggests that there is a much simpler way to analyse the latter. That is, instead of using convoluted realizations of the $N=2$ superconformal algebra like (2.12)-(2.14), and complicated expressions like (4.1) to describe the ground ring, it might be better adopt the equivalent, standard realization (3.7) [32] of the $N=2$ superconformal minimal models, where, for example, chiral ring elements can be represented more simply by vertex operators of the form $x^{j}=$ $e^{\frac{j}{\sqrt{2 t}}\left(\phi_{2}+i \phi_{1}\right)}$.

$\star$ The ghost number two extra states are dropped since they violate Seiberg's bound (4.10). 


\section{2. $W_{3}$-gravity coupled to $c \leq 2 W_{3}$-matter}

We showed in sections 2 and 3 that the (improved) BRST current of $W_{3}$ strings (2.27) is part of an extended $N=2$ superconformal $W_{3}$ algebra. This means that, as for ordinary strings, the physical operators of $W_{3}$ gravity coupled to matter constitute a chiral ring, $\mathcal{R}$.

In ordinary strings with $c^{M} \leq 1$ there are, typically, infinitely many physical operators [37-39], at arbitrary ghost numbers. One certainly expects to find a similar structure of extra states for $W_{3}$-strings, though, as yet, there exists no rigorous, general proof for the existence of such states. What we will do, therefore, is to prove the existence of some of these states by constructing them explicitly ${ }^{\dagger}$, guided by analogy to $c^{M} \leq 1$ strings. However, in order to avoid disrupting the line of thought with technicalities, we will relegate the explicit construction to Appendices A and B, and use only the results here.

We find that the analogues of tachyons and ground ring elements of ordinary gravity are physical operators $\mathcal{O}^{(Z)}, Z=A, B, \ldots, F$, with ghost numbers ranging from to 0 to 3. (As in ordinary gravity, some of these states only exist at discrete values of the momenta.) In particular, the tachyons, which have ghost number three, are denoted by $\mathcal{O}^{(A)}$, and the ground ring elements that have vanishing ghost number are denoted by $\mathcal{O}^{(F)}$. The ground ring is generated by four elements, which can be represented as:

$$
\begin{array}{rlrl}
x_{1} & \equiv \mathcal{O}_{1,1 ; 2,1}^{(F)}, & \gamma_{1}^{0} \equiv \mathcal{O}_{1,2 ; 1,1}^{(F)}, \\
x_{2} \equiv \mathcal{O}_{1,1 ; 1,2}^{(F)}, & \gamma_{2}^{0} \equiv \mathcal{O}_{2,1 ; 1,1}^{(F)} .
\end{array}
$$

Their explicit form is given in Appendix B. The generators have charges $q_{x_{i}}=i / t$, $q_{\gamma_{i}^{0}}=i$ with respect to the $N=2, U(1)$ current (2.29). In analogy to ordinary gravity, one finds that $x_{1}, x_{2}$ are primary with respect to the $N=2$ super- $W_{3}$ algebra, while $\gamma_{1}^{0}$ and $\gamma_{2}^{0}$ are not primary (except when $t=1$, which corresponds to $c^{M}=2$ matter coupled to $W_{3}$ gravity).

$\dagger$ Extra states for a different class of $W_{3}$ strings have recently been obtained in 47]. 
The associated reduced $N=2$ superconformal models that were obtained via hamiltonian reduction in section 3 , are defined by employing the full set of screening operators. This set of screening operators (3.20) consists of bosonic screeners, $\oint S_{M}^{ \pm}\left(\alpha_{i}\right), i=1,2$ and $\oint S_{L}^{ \pm}\left(\alpha_{2}\right)$, as well as of one fermionic screener:

$$
\widetilde{\mathcal{Q}}=\oint \frac{d z}{2 \pi i}\left[b_{2}-\sqrt{2} b_{1} \partial \phi_{M, 2}-2 i \sqrt{\frac{t}{3}} b_{1} b_{2} c_{2}+i \frac{(3+t)}{2 \sqrt{3 t}}\left(\partial b_{1}\right)-\frac{t}{3}\left(\partial b_{1}\right) b_{1} c_{2}\right] V_{12 ; 11}^{L} V_{12 ; 11}^{M}
$$

All these screeners (anti-)commute with $\mathcal{Q}_{B R S T}$.

It is straightforward to check that, up to factors and exact terms:

$$
\gamma_{1}^{0}=\left\{\widetilde{\mathcal{Q}}, \Gamma_{1}\right\}
$$

where

$$
\begin{aligned}
& \Gamma_{1}=\left(\frac{3 i t\left(9 t^{2}-2 t^{3}+1\right)}{2(1+t)} \sqrt{\frac{2}{3}} \partial \phi_{L, 1}-i \frac{t\left(5 t+4 t^{2}-5\right)}{\sqrt{2}} \partial \phi_{L, 2}\right)\left[b_{1}\left(\partial c_{2}\right) c_{2}-c_{1}\right] \\
& +t\left(1+t+t^{2}\right)\left(\sqrt{6} \partial \phi_{M, 1}+2 \sqrt{2} \partial \phi_{M, 2}\right)\left[c_{1}+b_{1}\left(\partial c_{2}\right) c_{2}\right] \\
& +\left(\frac{t(t-9)}{4} \partial \phi_{L, 1}^{2}+\frac{3 t\left(2 t^{3}-9 t^{2}-1\right)}{\sqrt{3}(1+t)} \partial \phi_{L, 1} \partial \phi_{L, 2}-\frac{t\left(19 t+16 t^{2}-11\right)}{4} \partial \phi_{L, 2}{ }^{2}-\frac{t(t-9)}{4} \partial \phi_{M, 1}{ }^{2}\right. \\
& +\frac{6 t\left(1+t+t^{2}\right)}{\sqrt{3}} \partial \phi_{M, 1} \partial \phi_{M, 2}-\frac{t}{4}\left(7+17 t+16 t^{2}\right) \partial \phi_{M, 2}^{2}-\frac{\sqrt{2 t}}{4}(t+1)(t-9)\left(\partial^{2} \phi_{L, 1}\right) \\
& +\frac{\sqrt{6 t}}{4}\left(3+t+4 t^{2}\right)(3 t-1)\left(\partial^{2} \phi_{L, 2}\right)-i \frac{\sqrt{2 t}}{4}(t-1)(t-3)\left(\partial^{2} \phi_{M, 1}\right) \\
& \left.-i \frac{\sqrt{6 t}}{4}(1+3 t)(t-1)(3+4 t)\left(\partial^{2} \phi_{M, 2}\right)\right) c_{2}+\left(\frac{3 \sqrt{2 t}}{4}\left(2 t^{3}-9 t^{2}-1\right) \partial \phi_{L, 1}\right. \\
& \left.-\frac{3 \sqrt{6 t}}{4}(1+3 t)(3 t-1) \partial \phi_{L, 2}-\frac{3 i \sqrt{2 t}}{2}(t-1)\left(1+t+t^{2}\right) \partial \phi_{M, 1}\right)\left(\partial c_{2}\right) \\
& +18 i(1-t)\left(1+t+t^{2}\right) \sqrt{\frac{t}{3}}\left[b_{1}\left(\partial^{2} c_{2}\right) c_{2}+\left(\partial b_{1}\right)\left(\partial c_{2}\right) c_{2}\right] \\
& +\frac{t}{2}\left(1-9 t-8 t^{2}\right)\left[\left(\partial b_{1}\right) c_{1} c_{2}+b_{1} c_{1}\left(\partial c_{2}\right)+3 b_{2}\left(\partial c_{2}\right) c_{2}\right]
\end{aligned}
$$

is a string- $B R S T$ invariant operator. Once again this means that $\gamma_{1}^{0}$ is exact with respect to the complete $N=2$ superconformal Felder $B R S T$ charge. While it is not so simple to explicitly demonstrate, it also turns out that $\gamma_{2}^{0}$ is similarly exact. The easiest way to see that it must be exact follows from the connection with the topologically twisted Kazama-Suzuki models that will be discussed below. Consequently, exactly as in ordinary gravity, the ring generators $\gamma_{i}^{0}$ are not physical operators in the reduced $N=2$ superconformal models. However, by requiring equivariance, these 
operators become physical and thus the $N=2$ models describe $W_{3}$-matter coupled to $W_{3}$-gravity.

We noted in section 2 that for the special $W_{3}$ minimal models $\mathcal{M}_{1, q=k+3}^{(3)}$ coupled to $W_{3}$ gravity, the anomaly of the topological twisted $N=2$ superconformal algebra is equal to $c^{N=2}=\frac{6 k}{k+3}$. This is the same as the central charge of the well-known $N=2$ superconformal models of Kazama and Suzuki [8], based on cosets $S U(3)_{k} / U(2)$, which happen to have the $N=2$ superconformal $W_{3}$ algebra as their chiral algebra. This implies that for integer $t \geq 3$, the reduced models are identical to the topological minimal $W_{3}$ models, whose chiral rings are given by certain polynomials in $x_{1}$ and $x_{2}$ and have been thoroughly studied and are well understood [36,48]. If one requires equivariance then the generators $\gamma_{1}^{0}$ and $\gamma_{2}^{0}$ also become physical, and thus the full chiral ring of the $\mathcal{M}_{1, q=k+3}^{(3)}$ model coupled to $W_{3}$ gravity is

$$
\mathcal{R}=\left\{\left(x_{1}\right)^{j_{1}}\left(x_{2}\right)^{j_{2}}\left(\gamma_{1}^{0}\right)^{n_{1}}\left(\gamma_{2}^{0}\right)^{n_{2}} \mid 0 \leq j_{1}+j_{2} \leq k \equiv t-3, n_{1}, n_{2} \geq 0\right\}
$$

One may suspect that such " $W$-gravitationally extended" rings of the Kazama-Suzuki models describe the coupling of topological minimal $W_{3}$ models to some form of topological $W_{3}$ gravity. However, the precise connection to the kind of topological $W$-gravity that is discussed in [49] is unclear, and in order to more firmly establish these statements, a deeper analysis is required.

There are other physical operators with non-vanishing ghost numbers and these appear to fall into modules of the ground ring. (Note that for the $\mathcal{M}_{1, q}^{(3)}$ models, the embedding diagram degenerates to a hexagon, and hence there is only a finite number of extra ghost sectors). Adopting a slightly different notation as in Appendix A, one thus would have, up to $B R S T$ exact pieces:

$$
\mathcal{O}_{s_{1}+t r_{1}, s_{2}+t r_{2}}^{(Z)} \sim\left(x_{1}\right)^{s_{1}}\left(x_{2}\right)^{s_{2}}\left(\gamma_{1}^{0}\right)^{r_{1}}\left(\gamma_{2}^{0}\right)^{r_{2}} \mathcal{P}^{(Z)}, \quad Z=A, B, \ldots, F
$$

(If one uses a free field formulation, one must remember to perform appropriate screenings or choose appropriate representatives of the operators $x_{i}$ and $\gamma_{i}^{0}$ in this equation.) 
The operators $\mathcal{P}^{(Z)}$ denotes a collection of puncture operators, which can be represented as ${ }^{\star}$ :

$$
\begin{aligned}
\mathcal{P}^{(A)} & =c_{1}\left(\partial c_{2}\right) c_{2} V_{0,0 ; 1,1}^{L} V_{0,0 ;-1,-1}^{M} \\
\mathcal{P}^{(B)} & =\left[c_{1} c_{2}+\frac{i}{6} \sqrt{\frac{3}{t}}(3-t)\left(\partial c_{2}\right) c_{2}\right] V_{1,0 ; 1,1-t}^{L} V_{1,0 ; 1, t-2}^{M} \\
\mathcal{P}^{(C)} & =\left[c_{1} c_{2}+\frac{i}{6} \sqrt{\frac{3}{t}}(t-3)\left(\partial c_{2}\right) c_{2}\right] V_{1,1 ; 1-t, 1}^{L} V_{1,1 ; t-2,1}^{M} \\
\mathcal{P}^{(F)} & =\mathbf{1} .
\end{aligned}
$$

One can check that these puncture operators have vanishing quantum numbers under the twisted $N=2$ super- $W_{3}$ algebra, so that they should represent equivalent copies of the vacuum. This then would imply, assuming that the ground ring acts faithfully on these vacuum sectors, that the operators $\mathcal{O}^{(Z)}, Z=A, B, \ldots, F$, represent the same chiral ring elements.

The (unperturbed) three-point function on the sphere can be written as

$$
\left\langle\mathcal{P}^{(B)} \mathcal{P}^{(A)}\left(\mathcal{P}^{(A)} \mathcal{O}_{1,1 ; t-2,1}^{(F)}\right)\right\rangle=1
$$

and this is precisely what one would have for topological $W_{3}$ gravity coupled to topological $W_{3}$ matter. In particular, all zero modes of the ghosts on the $W_{3}$-sphere are saturated. Above, $\mathcal{O}_{1,1 ; t-2,1}^{(F)} \sim\left(x_{2}\right)^{t-3}$ denotes the top element of the chiral ring of the topological matter model.

From section 3 we know that the $B R S T$ current for $W_{n}$-gravity coupled to $W_{n^{-}}$ matter, for general $n$, is part of an $N=2$ super- $W_{n}$ algebra. We therefore expect that the results of this sections will generalize to arbitray $n$. In particular, the "topological $W_{n}$-strings" with $t=k+n, k=0,1,2 \ldots$, should correspond to the Kazama-Suzuki models based on $S U(n)_{k} / U(n-1)$ coupled to (some form of) topological $W_{n}$-gravity [49], as conjectured in [25]. The "chiral ring," $\mathcal{R}$, will be generated by $x_{i}$ and $\gamma_{i}^{0}$, $i=1, \ldots, n-1$, where the $x_{i}$ are the usual chiral primary fields with $N=2, U(1)$ charges $q_{x_{i}}=i / t$ and the $\gamma_{i}^{0}$ have $N=2, U(1)$ charges $q_{\gamma_{i}^{0}}=i$, but are non-primary in the $N=2$ superconformal algebra.

* We have not been able to construct the operators $\mathcal{P}^{(D)}$ and $\mathcal{P}^{(E)}$ since they do not seem to have a form that fits a simple ansatz. 


\subsection{The cosmological constant and $G / G$ models}

Another approach to "topological $W_{n}$-strings" has been made by considering $G / G$ coset models [12,13]. This approach is probably equivalent to ours, and a direct connection can be made as follows. It was observed in [50,51,10] that topological $G / G$ models (without coupling to topological gravity) are closely related to the topologically twisted Kazama-Suzuki models based on $G / H$. In particular, if one makes a very specific, supersymmetry preserving perturbation of the twisted Kazama-Suzuki model one obtains precisely the topological $G / G$ models [10]. This perturbing operator is of the form $\psi=G_{-1 / 2}^{-} \bar{G}_{-1 / 2}^{-} \Phi$, where $\Phi(z)$ is a specific chiral primary field of dimension $h=\frac{k}{2(k+g)}$. In our formulation of the $N=2$ superconformal models, where $G / H=S U(n) / U(n-1)$, the operator $\psi$ can be represented by

$$
\psi=S_{L}^{-}\left(\alpha_{1}\right) \equiv e^{\frac{1}{\sqrt{t}} \alpha_{1} \cdot \phi_{L}}
$$

This operator describes precisely the cosmological constant perturbation, if one interprets the Kazama-Suzuki models as theories of $W$-matter coupled to $W$-gravity. It has a structure very similar to the other operators $S_{L}^{-}\left(\alpha_{i}\right), i=2, \ldots, n-1$, which have vanishing $N=2$ superconformal quantum numbers (in contrast to $\psi$ ), and which appear as screening operators in the reduced $N=2$ superconformal models.

From the perspective of the $N=2$ superconformal models, $\psi$ is a relevant, supersymmetry preserving operator, but has non-vanishing $N=2, U(1)$ charge. As a result, we expect the $G / G$ models to exhibit merely $N=2$ supersymmetry, and not $N=2$ superconformal invariance. Moreover, because of the close relationship of $\psi$ to the screening currents, there will in fact be an $N=2$ supersymmetric (but not superconformal) $W$-algebra at generic values of the cosmological constant. This is precisely because the perturbation by $\psi$ of the Kazama-Suzuki models leads to quantum integrable $N=2$ Toda theories with conserved currents associated with supersymmetric $W$-algebras [52,10]. Indeed, when the model is coupled to $W$-gravity, the proof that the the top components of the $N=2$ super- $W$ multiplets (2.33) provide "off-critical" conserved $W$-charges is exactly the same as it is for the perturbed Kazama-Suzuki models.

Putting it slightly differently, if one introduces a cosmological constant perturbation to the $W$-string, then the perturbation breaks $N=2$ superconformal invariance 
down to a massive $N=2$ supersymmetry algebra. As a result, the string $B R S T$ charge receives a very simple, "off-critical" perturbative correction, exactly as in [53,54]. This superalgebra will have a $W$-extension arising from the infinite number of conserved quantities of the underlying quantum-integrable model.

\section{Final comments and speculations}

From the results of sections $2-4$, it is evident that the occurence of $N=2$ superconformal symmetry is rather general in matter models coupled to gravity and in $W$-extensions thereof. The utility of this observation will depend greatly upon the model under consideration. In particular, the quality of what one learns depends upon whether the underlying $N=2$ super-W model is minimal or not. For the 26-dimensional bosonic string, the $N=2$ structure will probably not improve our understanding significantly, howevever, for the minimal models $\mathcal{M}_{1, q}$ coupled to $W_{n^{-}}$ gravity with $q=k+n$, the $N=2$ structure provides valuable new insight.

The connection between topological $W$-strings and Kazama-Suzuki models raises a number of new questions. For example, the Kazama-Suzuki models possess [8] duality symmetries that imply that models based upon the coset

$$
\frac{S U(m+n)_{k}}{S U(m)_{k+n} \times S U(n)_{k+m} \times U(1)}
$$

yield the same $N=2$ superconformal model under all permutations of $m, n$ and $k$. If this duality is also a symmetry for the corresponding $W$-string, it would, amongst other things, imply the equivalence of topological $W_{n+1}$-strings based on $\frac{S U(n+1)_{1}}{S U(n)_{2} \times U(1)}$ with ordinary topological strings based on $\frac{S U(2)_{n}}{U(1)}$. In addition such a duality symmetry suggests that one can generalize the reduction procedure of section 3 to KazamaSuzuki models, $G / H$, that are more general than those based on $\mathbb{C} \mathbb{P}_{n}$. In this context it is interesting to note that for a given group $G$, the structure of the twisted $N=2$ superconformal theory is essentially determined by the choice the $N=2, U(1)$ current $^{\star}$. In our formulation this is reflected [50,51] in the various possible choices for the fundamental weight, $\lambda_{1}$, of $G$ in (the appropriate generalization of) the $U(1)$ current $(2.29)$.

^ This is only strictly true for models based on hermitian, symmetric spaces. 
Over the past year it has also become apparent that if one formulates topological matter models in an appropriate manner, one can also obtain the coupling to topological gravity. For example, topological $G / G$ models [11,12,10 have a finite number of topological "matter" fields. However, if the $G / G$ models are formulated using the entire gauge, ghost and associated BRST structure [13], it appears that one can extract the $W$-gravity descendants. It has also been proposed that one can obtain topological minimal matter coupled to gravity from the Landau-Ginzburg potentials $W(x)=x^{k+2}$ of the topological minimal models, by careful regularization and proper treatment of contact terms [55].

Our results can also be interpreted in the same spirit: We find that the free field realization of twisted $N=2$ superconformal minimal models contains the ingredients needed to describe the coupling to topological gravity. That is, the choice of whether or not to use the extra component, $\widetilde{\mathcal{Q}}$, of $N=2$ Felder BRST operators (or, equivalently, whether or not to impose that the requirement that cohomology be equivariant), corresponds precisely to describing the topological minimal matter theories either with or without the coupling to topological gravity. We have also seen that this generalizes to topological $W$-strings. We find that each Landau-Ginzburg variable, $x_{i}$, is paired with another non-primary, chiral ring element, $\gamma_{i}^{0}$. Each such $\gamma_{i}^{0}$ is supposed to correspond to a $W$-gravitational dressing of the topological matter. Based on the proposals in [55] it is tempting to suggest that these $\gamma_{i}^{0}$ 's could be obtained directly from the Landau-Ginzburg potentials [36,48,54] of the Kazama-Suzuki models through some form of contact terms induced by integration over " $W$-moduli".

The foregoing pairing of the matter fields, $x_{i}$, and the $W$-gravity dressing operators, $\gamma_{i}^{0}$, is not unexpected from the general structure of Kazama-Suzuki models. It is known that in the $\mathbb{C P}_{n}$ models there is a duality between the chiral ring, $\mathcal{R}$, and the chiral algebra, $\mathcal{A}[30$. Specifically, there is a one-to-one correspondence between the generators, $x_{i}$, of $\mathcal{R}$ and the $N=2$ supermultiplets, $\mathcal{V}_{s}(2.33)$, of $W$-generators in $\mathcal{A}$. In a theory of topological $W$-gravity [49], one expects a $\gamma$-ghost for each generator of the chiral algebra, and therefore one sees that each $x_{i}$ will be paired with a $\gamma_{i}^{0}$.

Finally, we note that the presence of an $N=2$ superconformal algebra automatically implies the existence of an infinite number of higher spin bosonic operators in the chiral algebra. That is, in addition to any independent super- $W$ generators, there 
are composite operators made from $G^{+}(z), G^{-}(z), J(z)$ and $T(z)$. It was shown in [56] that any $N=2$ superconformal theory with $c^{N=2} \geq 3$ can be thought of as a coset

$$
\frac{S U(1,1) \times S O(2)}{U(1)}
$$

in which the supercharges are represented by $G^{ \pm}(z)=\psi^{ \pm}(z) e^{ \pm i \alpha X(z)}$, where the $\psi^{ \pm}(z)$ are parafermions of $S U(1,1) / U(1)$, and $X(z)$ is defined by $J(z)=i \sqrt{\frac{c}{3}} \partial X(z)$. The chiral algebra of $S U(1,1) / U(1)$ can be formed from parafermion bilinears and form an infinite $W$-algebra [57]. For minimal models with $c^{N=2}=3 k /(k+2)$ there is a finite $W_{k}$-algebra made from composites of $G^{+}(z), G^{-}(z), J(z)$ and $T(z)$. It is therefore tempting to suggest that the previously known extended $W$-symmetries of topological matter coupled to topological gravity should be related to the foregoing composite super $W$-generators. One should however note that the coset formulation (5.2) is not exactly that of the black-hole metric [58] since the $S U(1,1)$ structure of the black-hole involves only Liouville and matter fields, and is orthogonal to the ghost Hilbert space. The $U(1)$ factors in (5.2) involve mixings with the ghost sector. However it seems likely that the extended $W$-symmetries of the black-hole metric should have some nice formulation in terms of the enveloping algebra of the $N=2$ superconformal algebra.

\section{Acknowledgements}

We thank P. Bouwknegt, K. Li, B. Gato-Rivera, H. Ooguri, K. Pilch and C. Vafa for discussions. W.L. also thanks John Schwarz for generous support of his visit to Caltech, during which time this paper was completed. We finally thank K. Thielemans for providing us with the Mathematica package OPEdefs [59], which was essential for many of the computations. M.B. is partially supported by NSF grant PHY 87/14654 and by Packard Fellowship 89/1624, D.N. and N.P.W. are supported in part by funds provided by the DOE under grant No. DE-FG03-84ER40168. N.P.W. was also partially supported by a fellowship from the Alfred P. Sloan Foundation, and is grateful to the Theory Division at CERN for its hospitality and support during the early stages of this work. 


\section{Appendix A. Extra states for non-critical $W_{3}$ strings}

In section 4 we generalized some of our observations to $W_{3}$-gravity coupled to $W_{3}$ matter. As a comprehensive study of the physical operators in this kind of theories has not, as yet, appeared in the literature, we will present below some remarks on the construction of such operators, as well as some explicit results.

In ordinary strings with $c^{M} \leq 1$ and for a given matter primary field $\Phi_{r, s}$, the spectrum of extra states is determined by the structure of the embedding diagram of null states over $\Phi_{r, s}$ [37,38]: each dot of this diagram denotes a null state in the matter theory, and typically gives rise to a physical state in the matter plus gravity system. The ghost number is given by the number of steps it takes to reach the given dot from the top of the diagram. (The top of the diagram corresponds to the primary field $\Phi_{r, s}$ itself, and leads to the standard tachyon in the matter plus gravity system.) One thus obtains, generically, infinitely many ghost sectors. In some cases (for example, for the fields in minimal matter models $\mathcal{M}_{1, q}$ ), the Felder resolution becomes finite [60] and the relevant part of the diagram consists of just two points, which can be associated with the standard tachyon and a ground ring element. These two kinds of operators are the most generic ones in that they exist for all completely degenerate $\Phi_{r, s}$ of the models $\mathcal{M}_{p, q}$ (including the $c^{M}=1$ model where $p=q$ ).

It is the analogue of this finite subset of extra states (with zero or negative relative $^{\star}$ ghost numbers) in which we are presently interested. Specifically, we will associate such extra states with the "generic" null states in $W_{3}$ minimal models, $\mathcal{M}_{p, q}^{(3)}$. The relevant part of the embedding diagram looks like a hexagon whose corners are associated to the elements of the Weyl group $W$ of $S U(3)$ [61] (typically, the hexagon is part of an infinitely extended diagram, whose dots correspond to the affine Weyl group). For later reference, we will labels these corners by $A, B, \ldots, F$, and denote a generic such label by $Z$ :

$\star$ With "relative ghost numbers" we mean ghost numbers relative to the ghost number of the tachyon. We understand that there certainly should exist closely related states at positive relative ghost numbers, but we will not consider these here. 


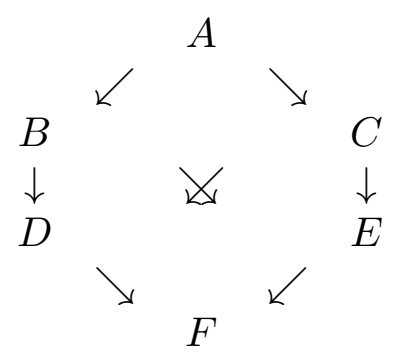

The arrows correspond to embeddings of Verma modules. Label $A$ corresponds to some primary field $\Phi_{r_{1}, r_{2} ; s_{1}, s_{2}}$ (in standard notation), and labels $B-F$ correspond to null states over this primary. The levels $\ell(Z)$ of these states are given by:

\begin{tabular}{c|c|c} 
sector & element & level \\
$\mathrm{Z}$ & $w(Z) \in W$ & $\ell(Z)$ \\
\hline $\mathrm{A}$ & 1 & 0 \\
$\mathrm{~B}$ & $w_{1}$ & $r_{1} s_{1}$ \\
$\mathrm{C}$ & $w_{2}$ & $r_{2} s_{2}$ \\
$\mathrm{D}$ & $w_{2} w_{1}$ & $r_{1} s_{1}+r_{2} s_{1}+r_{2} s_{2}$ \\
$\mathrm{E}$ & $w_{1} w_{2}$ & $r_{1} s_{1}+r_{1} s_{2}+r_{2} s_{2}$ \\
$\mathrm{~F}$ & $w_{2} w_{1} w_{2}$ & $\left(r_{1}+r_{2}\right)\left(s_{1}+s_{2}\right)$
\end{tabular}

This association with Weyl group elements can be made explicit by employing a free field formulation of the $W_{3}$ minimal models, as described in section 2. Label $A$ will then be associated with the standard Fock space, $\mathcal{F}_{r 1, r 2 ; s 1, s 2}$, with $\sum r_{i} \leq p-1$ and $\sum s_{i} \leq q-1$. The Weyl group can be taken to act on the vertex operators (2.5) as:

$$
w \in W: \quad \alpha_{r_{i} ; s_{i}}^{M} \rightarrow \alpha_{r_{i} ; w * s_{i}}^{M} \equiv \alpha_{+} \sum_{j}\left(r_{j}-1\right) \lambda_{j}+\alpha_{-} w\left(\sum\left(s_{j}-1\right) \lambda_{j}\right) .
$$

The null states are then given by applying screening operators to the transformed vertex operators, which map back into null states in the standard Fock space. It is easy to check that under (A.2), the dimension of vertex operators (2.5) changes precisely by $\ell(w(Z))$, as given in the above table. 
In analogy to usual gravity coupled to matter, we expect in $W$-gravity coupled to matter extra $B R S T$ states to arise for each dot of the hexagon, with ghost number $g$ given by the number of steps from the top of the the diagram to the given dot, that is, by the length of the Weyl group element. The top of the diagram, sector $A$, should describe the standard tachyons. These operators have the form

$$
\mathcal{O}_{r 1, r 2 ; s 1, s 2}^{(A)}=c_{1}\left(\partial c_{2}\right) c_{2} V_{r 1, r 2 ;-s 1,-s 2}^{L} V_{r 1, r 2 ; s 1, s 2}^{M}
$$

and were shown in [25] to be indeed non-trivial elements of the $B R S T$ cohomology. They have ghost number equal to $g=3$, and it is clear from the hexagon that the extra states that we are seeking have ghost numbers $g=2,1,0$. In particular, the bottom of the diagram will correspond to ground ring elements with vanishing ghost number.

There is actually an ambiguity in writing the tachyonic (and other) operators, as the $L_{0}$ and $W_{0}$ eigenvalues of $V^{M}$ and $V^{L}$ are invariant under certain Weyl transformations. The Weyl group action in question, denoted by $\Sigma$, is different from the Weyl group action $W$, defined above. For $\Sigma$ we define:

$$
\begin{aligned}
\sigma_{L} \in \Sigma_{L}: & \alpha_{r_{i} ; s_{i}}^{L} \rightarrow \alpha_{\sigma_{L} * r_{i} ; \sigma_{L} * s_{i}}^{L} \equiv \sigma_{L}\left(\alpha_{r_{i} ; s_{i}}^{L}-\beta_{0} \rho\right)+\beta_{0} \rho \\
\sigma_{M} \in \Sigma_{M}: & \alpha_{r_{i} ; s_{i}}^{M} \rightarrow \alpha_{\sigma_{M} * r_{i} ; \sigma_{M} * s_{i}}^{M} \equiv \sigma_{M}\left(\alpha_{r_{i} ; s_{i}}^{M}-\alpha_{0} \rho\right)+\alpha_{0} \rho
\end{aligned}
$$

That is, a physical operator can typically be represented by six different vertex operators. In the matter sector, these six copies are equivalent, and we can always choose the standard labels, $\sum r_{i} \leq p-1$ and $\sum s_{i} \leq q-1, r_{i} \geq 1, s_{i} \geq 1$, to represent a matter primary by a vertex operator.

The six copies in the Liouville sector, on the other hand, are not on equal footing. This is similar to ordinary gravity where the Weyl group is $\mathbb{Z}_{2}$. It is indeed well-known 62 that in usual gravity there are two possible Liouville dressings for a given matter primary $\Phi_{r, s}$; one is given by $e^{\alpha_{r ;-s}^{L} \phi_{L}}$ and the other one by $e^{\alpha_{-r ; s}^{L} \phi_{L}}$. These dressings are, however, not equivalent: the second of the dressings violates Seiberg's condition (4.10), which requires that the Liouville momenta must satisfy: $\alpha^{L}<\frac{1}{\sqrt{2}} \beta_{0}$. That means that this latter dressing should be discarded. This is extra physical input, coming from the destabilizion of Riemann surfaces by macroscopic loops [42]. From representation theory alone, there is nothing wrong with states with the "wrong" 
dressing. They are related to the dual resolution in the Felder complex, and give rise to extra states with zero and positive relative ghost number. Moreover, the matching of scaling dimensions of operators in matter plus gravity theories with those of matrix models also requires restriction to operators that satisfy Seiberg's condition (4.10) [42]. Furthermore, we introduced in section 2 certain $N=2, U(1)$ currents that involve the Liouville field explicitly. The $U(1)$ charge of Liouville vertex operators will thus in general depend on $\Sigma_{L}$ transformations, and this means that the Liouville dressings cannot be equivalent.

Unfortunately, we do not rigorously know what the analogue of Seiberg's bound is for $W$-gravity. But we note that $\alpha_{L}=\beta_{0} \rho$ is the fixed point of the Weyl transformations (A.4) and it is this what should correspond to Seiberg's bound (4.10). We will therefore assume that the Liouville momentum must lie within (and not on) the boundary of a particular Weyl chamber shifted by $\beta_{0} \rho$. This Weyl chamber corresponds precisely to the choice that we made in the representation (A.3) of the tachyons. (Our assumption will be justified a posteriori, in that we will indeed find non-trivial cohomology at the correct (negative relative) ghost numbers. A further consistency check is as follows. For the particular weight basis: $\lambda_{1}=\left(\frac{1}{\sqrt{2}}, \frac{1}{\sqrt{6}}\right), \lambda_{2}=\left(0, \frac{2}{\sqrt{6}}\right)$, it can be inferred from 18 that $\phi_{L, 1}$ can be viewed as the "usual" Liouville field as part of $W$-gravity. Our choice of tachyon representative indeed satisfies Seiberg's condition for the first components of Liouville momenta: $\left(\alpha_{r_{i},-s_{i}}^{L}\right)_{1}<\frac{1}{\sqrt{2}} \beta_{0}$ for labels in the standard range.)

As in usual theories of gravity coupled to matter, we expect that the Liouville dressing of an extra state corresponds to the dressing of a null state over a matter primary. Thus, the extra state should be given by a polynomial, $X$, in the ghosts and in $\partial \phi_{L, M}$, of appropriate conformal dimension $\ell(Z)$ and ghost number $g$, times a vertex operator piece $\mathcal{V}$ :

$$
\mathcal{O}_{r 1, r 2 ; s 1, s 2}^{(Z)}=X_{-\ell(Z)}^{(Z)} c_{1}\left(\partial c_{2}\right) c_{2} \mathcal{V}_{r 1, r 2 ; s 1, s 2}^{(Z)}
$$

The Liouville dressing can be obtained by Weyl transformations (A.2) $w_{L} \in W_{L}$ acting on the Liouville part of the tachyons (A.3):

$$
\mathcal{V}_{r 1, r 2 ; s 1, s 2}^{(Z)} \equiv V_{r_{1}, r_{2} ; w(Z) *\left(-s_{1}\right), w(Z) *\left(-s_{2}\right)}^{L} V_{r 1, r 2 ; s 1, s 2}^{M}
$$


From the first table we have

$$
h\left(\mathcal{V}^{(Z)}\right)=4-\ell(Z)
$$

so that all operators $\mathcal{O}_{r 1, r 2 ; s 1, s 2}^{(Z)}$ have vanishing dimensions. More explicitly, the precise Liouville dressings are:

\begin{tabular}{c|c|c} 
sector & Liouville dressing & ghost number of \\
$\mathrm{Z}$ & $V_{r_{1}, r_{2} ; w(Z) *\left(-s_{1}\right), w(Z) *\left(-s_{2}\right)}^{L}$ & $X^{(Z)}$ \\
\hline $\mathrm{A}$ & $V_{r_{1}, r_{2} ;-s_{1},-s_{2}}^{L}$ & 0 \\
$\mathrm{~B}$ & $V_{r_{1}, r_{2} ; s_{1},-s_{1}-s_{2}}^{L}$ & -1 \\
$\mathrm{C}$ & $V_{r_{1}, r_{2} ;-s_{1}-s_{2}, s_{2}}^{L}$ & -1 \\
$\mathrm{D}$ & $V_{r_{1}, r_{2} ;-s_{2}, s_{1}+s_{2}}^{L}$ & -2 \\
$\mathrm{E}$ & $V_{r_{1}, r_{2} ; s_{1}+s_{2},-s_{1}}^{L}$ & -2 \\
$\mathrm{~F}$ & $V_{r_{1}, r_{2} ; s_{2}, s_{1}}^{L}$ & -3
\end{tabular}

All that remains is to make an ansatz for $X^{(Z)}$ with the correct quantum numbers and to solve for $\left[\mathcal{Q}_{B R S T}, \mathcal{O}_{r 1, r 2 ; s 1, s 2}^{(Z)}\right\}=0$, where the $B R S T$ current is given by $(2.26)^{\star}$. This yields a highly overdetemined set of equations, and the fact that we do find solutions shows that our assumptions about the structure of the extra states were correct. (One cannot exclude at this point the possibility that some solutions give $B R S T$-exact operators. We have checked, however, that our solutions indeed are non-trivial with respect to $(2.26)$.)

The simplest non-tachyonic operators that we find are those whose dimension of the vertex operator part does not depend on some combination of the $r_{i}, s_{i}$. For example, the dimension of $\mathcal{V}^{(B)}$ is independent of $s_{2} \equiv s$, and we find explicitly:

$$
\mathcal{O}_{1,1 ; 1, s}^{(B)}=\left[c_{1} c_{2}+\frac{i}{6} \sqrt{\frac{3}{t}}(3 t-2 s-1)\left(\partial c_{2}\right) c_{2}\right] V_{1,1 ; 1,-1-s}^{L} V_{1,1 ; 1, s}^{M}
$$

$\star$ Our representatives are such that $\mathcal{Q}_{B R S T}$ maps into vanishing null states. This faciliates the computation. 
Similarly,

$$
\mathcal{O}_{1,1 ; s, 1}^{(C)}=\left[c_{1} c_{2}-\frac{i}{6} \sqrt{\frac{3}{t}}(3 t-2 s-1)\left(\partial c_{2}\right) c_{2}\right] V_{1,1 ;-1-s, 1}^{L} V_{1,1 ; s, 1}^{M}
$$

Slightly more non-trivial is:

$$
\begin{aligned}
\mathcal{O}_{1,1 ; s, 2}^{(C)} & =\left[-\frac{i}{\sqrt{2 t}} c_{1}\left(\partial c_{2}\right)-\frac{1}{\sqrt{3}} c_{1} c_{2}\left[\partial \phi_{M, 2}+i \partial \phi_{L, 2}\right]\right. \\
& +\frac{1}{6 \sqrt{t}}\left(\partial c_{2}\right) c_{2}\left[(5+2 s-3 t) \partial \phi_{L, 2}-i(2 s-3 t-1) \partial \phi_{M, 2}\right] \\
& \left.+\frac{1}{\sqrt{6} t}(2 t-s-1) b_{1} c_{1}\left(\partial c_{2}\right) c_{2}+\frac{1}{\sqrt{6}}\left(\partial^{2} c_{2}\right) c_{2}\right] V_{1,1 ;-2-s, 2}^{L} V_{1,1 ; s, 2}^{M}
\end{aligned}
$$

Moreover, for sectors $D$ and $E$ we find that the simplest $B R S T$ invariant operators involve "screening" operators:

$$
\begin{aligned}
\mathcal{O}_{1,1 ; 1,1}^{(E)} & =\left[c_{1}-b_{1}\left(\partial c_{2}\right) c_{2}+\frac{i}{2} \sqrt{\frac{3}{t}}(t+1)\left(\partial c_{2}\right)+\frac{i}{\sqrt{2}} c_{2}\left[\partial \phi_{L, 2}+\sqrt{3} \partial \phi_{L, 1}\right]\right] S_{L}^{-}\left(\alpha_{2}\right) \\
\mathcal{O}_{1,1 ;-1,2}^{(E)} & =\left[c_{1}+b_{1}\left(\partial c_{2}\right) c_{2}-\frac{i}{2} \sqrt{\frac{3}{t}}(t-1)\left(\partial c_{2}\right)+\sqrt{2} c_{2} \partial \phi_{M, 2}\right] S_{M}^{-}\left(\alpha_{1}\right)
\end{aligned}
$$

where $S_{M}^{ \pm}\left(\alpha_{i}\right)$ are the matter screening operators (2.4) and $S_{L}^{ \pm}\left(\alpha_{i}\right)$ are their Liouville counterparts. Similarly, $\mathcal{O}_{1,1 ; 1,1}^{(D)} \sim S_{L}^{-}\left(\alpha_{1}\right)$, etc. Note that the foregoing are truly $B R S T$ invariant operators. From the descent equations (2.35) we infer that the screening operators themselves are $B R S T$ invariant up to total derivatives, so that $\oint S_{L, M}^{ \pm}\left(\alpha_{i}\right)$ are BRST invariant. Note also, as explained in more detail in section 4.3, that one of these operators (namely $\oint S_{L}^{-}\left(\alpha_{1}\right)$ ) is not a true screening operator, but represents a physical perturbation of the action.

More interesting, but also more complicated, are the ground ring operators. The dimension of $X^{(F)}$ grows rapidly with $r_{i}$ and $s_{i}$, and therefore its complexity dramatically increases at the same time. As a result we are only able to give the generators of the ground ring. We have collected the explicit expressions in Appendix B. Note that these operators exist for generic values of $t$, and in particular for $t=1$, which describes $c^{M}=2$ matter coupled to $W_{3}$ gravity. We expect that there should be a way of writing these ground ring generators, as well as general ground ring elements, more compactly in terms of Schur-like polynomials, but we have not succeeded in doing this. 
Note also that these operators are defined only up to $B R S T$ trivial pieces of the form $\left\{\mathcal{Q}_{B R S T}, b_{1} V^{L} V^{M}\right\}$. Using this freedom, one can remove the pieces proportional to $\partial^{2} c_{2}$ in the expressions for the ground ring generators, so that all generators are killed by the zero modes of the $b$ ghosts. However, we preferred to choose different representatives, namely operators that are primary with respect to the $N=2$ superconformal algebra (the expressions for $\gamma_{1}^{0}, \gamma_{2}^{0}$ are primary only for $t=1$ ). 


\section{Appendix B. Ground ring generators for $W_{3}$ gravity}

$$
\begin{aligned}
& x_{1} \equiv \mathcal{O}_{1,1 ; 2,1}^{(F)}= \\
& {\left[\frac{2}{3} \partial \phi_{L, 2}^{2}-\frac{i}{\sqrt{3}} \partial \phi_{L, 2} \partial \phi_{M, 1}-\frac{i}{3} \partial \phi_{L, 2} \partial \phi_{M, 2}-\frac{1}{\sqrt{3}} \partial \phi_{M, 1} \partial \phi_{M, 2}+\frac{1}{3} \partial \phi_{M, 2}^{2}\right.} \\
& -\frac{2}{3 t} b_{1} b_{2} c_{1} c_{2}-\frac{5 i}{3 t \sqrt{3 t}} b_{1} b_{2}\left(\partial c_{2}\right) c_{2}-\sqrt{\frac{2}{3 t}} \partial \phi_{L, 2} b_{1} c_{1}+i \frac{\left(21 t^{2}-5\right)}{12 t \sqrt{3 t}}\left(\partial b_{1}\right)\left(\partial c_{2}\right) \\
& +\left(i \frac{(t-5)}{3 \sqrt{2} t} \partial \phi_{L, 2}-\frac{2}{\sqrt{6}} \partial \phi_{M, 1}-\frac{\sqrt{2}}{3} \partial \phi_{M, 2}\right) b_{1}\left(\partial c_{2}\right)+i \frac{(4 t-3)}{2 \sqrt{3 t}} b_{1}\left(\partial^{2} c_{2}\right)+i \frac{1}{\sqrt{3 t}} b_{2} c_{1} \\
& +\left(\frac{i}{\sqrt{2 t}} \partial \phi_{M, 1}-\sqrt{\frac{2}{3 t}} \partial \phi_{L, 2}+\frac{i}{\sqrt{6 t}} \partial \phi_{M, 2}\right) b_{2} c_{2}+\frac{(9 t-5)}{6 t} b_{2}\left(\partial c_{2}\right)+\frac{i}{3 t \sqrt{3 t}}\left(\partial b_{1}\right) b_{1} c_{1} c_{2} \\
& +\frac{\left(9 t^{2}-24 t-5\right)}{18 t^{2}}\left(\partial b_{1}\right) b_{1}\left(\partial c_{2}\right) c_{2}+\frac{(3 t-1)}{6 t}\left(\partial b_{1}\right) c_{1}+\frac{i}{2} \sqrt{\frac{t}{3}}\left(\partial^{2} b_{1}\right) c_{2}-\sqrt{\frac{2 t}{3}}\left(\partial^{2} \phi_{L, 2}\right) \\
& +\left(i \frac{\sqrt{2}(t-1)}{6 t} \partial \phi_{L, 2}-\frac{(1+t)}{4 t} \sqrt{\frac{2}{3}} \partial \phi_{M, 1}-\frac{\sqrt{2}(1+t)}{12 t} \partial \phi_{M, 2}\right)\left(\partial b_{1}\right) c_{2}+\left(\partial b_{2}\right) c_{2} \\
& +b_{1} c_{2}\left(\frac{2 i}{3 \sqrt{3 t}} \partial \phi_{M, 2}{ }^{2}+\frac{2 i}{3 \sqrt{2}}\left(\partial^{2} \phi_{L, 2}\right)-\frac{4(t-1)}{3 \sqrt{2} t}\left(\partial^{2} \phi_{M, 2}\right)-i \frac{2}{3 \sqrt{3 t}} \partial \phi_{L, 2}{ }^{2}\right. \\
& \left.\left.-\frac{1}{3 \sqrt{t}} \partial \phi_{L, 2} \partial \phi_{M, 1}-\frac{1}{3 \sqrt{3 t}} \partial \phi_{L, 2} \partial \phi_{M, 2}-\frac{2 i}{3 \sqrt{t}} \partial \phi_{M, 1} \partial \phi_{M, 2}\right)+\frac{2 i(t-1)}{\sqrt{6 t}}\left(\partial^{2} \phi_{M, 2}\right)\right] \\
& \quad \times V_{1,1 ; 1,2}^{L} V_{1,1 ; 2,1}^{M}
\end{aligned}
$$

$$
\begin{aligned}
& x_{2} \equiv \mathcal{O}_{1,1 ; 1,2}^{(F)}= \\
& {\left[\frac{1}{3} \partial \phi_{L, 2}{ }^{2}+\frac{i}{\sqrt{3}} \partial \phi_{L, 1} \partial \phi_{M, 2}+\frac{i}{3} \partial \phi_{L, 2} \partial \phi_{M, 2}+\frac{2}{3} \partial \phi_{M, 2}^{2}-\frac{1}{\sqrt{3}} \partial \phi_{L, 1} \partial \phi_{L, 2}\right.} \\
& -i \sqrt{\frac{2}{3 t}} \partial \phi_{M, 2} b_{1} c_{1}+\frac{2}{3 t} b_{1} b_{2} c_{1} c_{2}-i \frac{5}{3 t \sqrt{3 t}} b_{1} b_{2}\left(\partial c_{2}\right) c_{2}-(1+t) \sqrt{\frac{2}{3 t}}\left(\partial^{2} \phi_{L, 2}\right) \\
& +\left(\frac{2 i}{\sqrt{6}} \partial \phi_{L, 1}+\frac{2 i}{3 \sqrt{2}} \partial \phi_{L, 2}-\frac{5+t}{3 \sqrt{2} t} \partial \phi_{M, 2}\right) b_{1}\left(\partial c_{2}\right)+i \frac{3+4 t}{2 \sqrt{3 t}} b_{1}\left(\partial^{2} c_{2}\right)+i \frac{1}{\sqrt{3 t}} b_{2} c_{1} \\
& +\left(\frac{1}{\sqrt{2 t}} \partial \phi_{L, 1}+\frac{1}{\sqrt{6 t}} \partial \phi_{L, 2}-i \sqrt{\frac{2}{3 t}} \partial \phi_{M, 2}\right) b_{2} c_{2}+\frac{5+9 t}{6 t} b_{2}\left(\partial c_{2}\right)+\frac{1+3 t}{6 t}\left(\partial b_{1}\right) c_{1} \\
& +\left(i \frac{(t-1)}{4 t} \sqrt{\frac{2}{3}} \partial \phi_{L, 1}+i \frac{\sqrt{2}(t-1)}{12 t} \partial \phi_{L, 2}-\frac{\sqrt{2}(1+t)}{6 t} \partial \phi_{M, 2}\right)\left(\partial b_{1}\right) c_{2}+i \frac{1}{3 t \sqrt{3 t}}\left(\partial b_{1}\right) b_{1} c_{1} c_{2} \\
& -\frac{\left(24 t+9 t^{2}-5\right)}{18 t^{2}}\left(\partial b_{1}\right) b_{1}\left(\partial c_{2}\right) c_{2}+i \frac{\left(21 t^{2}-5\right)}{12 t \sqrt{3 t}}\left(\partial b_{1}\right)\left(\partial c_{2}\right)+\left(\partial b_{2}\right) c_{2}+i \frac{1}{2} \sqrt{\frac{t}{3}}\left(\partial^{2} b_{1}\right) c_{2} \\
& +b_{1} c_{2}\left(\frac{2 i}{3 \sqrt{t}} \partial \phi_{L, 1} \partial \phi_{L, 2}-\frac{2 i}{3 \sqrt{3 t}} \partial \phi_{L, 2}{ }^{2}-\frac{1}{3 \sqrt{t}} \partial \phi_{L, 1} \partial \phi_{M, 2}-\frac{1}{3 \sqrt{3 t}} \partial \phi_{L, 2} \partial \phi_{M, 2}\right. \\
& \left.\left.+\frac{2 i}{3 \sqrt{3 t}} \partial \phi_{M, 2}^{2}+\frac{4 i(1+t)}{3 \sqrt{2} t}\left(\partial^{2} \phi_{L, 2}\right)-\frac{2}{3 \sqrt{2}}\left(\partial^{2} \phi_{M, 2}\right)\right)+2 i \sqrt{\frac{t}{6}}\left(\partial^{2} \phi_{M, 2}\right)\right] V_{1,1 ; 2,1}^{L} V_{1,1 ; 1,2}^{M}
\end{aligned}
$$




$$
\begin{aligned}
& \gamma_{1}^{0} \equiv \mathcal{O}_{1,2 ; 1,1}^{(F)}= \\
& {\left[\frac{3+5 t}{12} \partial \phi_{L, 2}{ }^{2}-\frac{(t-1)}{4} \partial \phi_{L, 1}{ }^{2}+\frac{1+t}{4} \partial \phi_{M, 1}{ }^{2}+\frac{2 i t}{3} \partial \phi_{L, 2} \partial \phi_{M, 2}-\frac{(5 t-3)}{12} \partial \phi_{M, 2}{ }^{2}\right.} \\
& -\frac{2 t^{2}}{3} b_{1} b_{2} c_{1} c_{2}-\frac{5 i t^{3}}{3 \sqrt{3 t}} b_{1} b_{2}\left(\partial c_{2}\right) c_{2}-\left((t+1) \sqrt{\frac{t}{6}} \partial \phi_{L, 2}+i(t-1) \sqrt{\frac{t}{6}} \partial \phi_{M, 2}\right) b_{1} c_{1} \\
& -i \frac{3+5 t^{2}}{6 \sqrt{2}}\left(\partial \phi_{L, 2}+i \partial \phi_{M, 2}\right) b_{1}\left(\partial c_{2}\right)+\frac{i}{2} \sqrt{\frac{t}{3}} b_{1}\left(\partial^{2} c_{2}\right)+i \frac{t^{2}}{\sqrt{3 t}} b_{2} c_{1}-\frac{\left(t^{2}-3\right)}{6}\left(\partial b_{1}\right) c_{1} \\
& -t^{2} \sqrt{\frac{2}{3 t}}\left(\partial \phi_{L, 2}+i \partial \phi_{M, 2}\right) b_{2} c_{2}-\frac{\left(5 t^{2}-9\right)}{6} b_{2}\left(\partial c_{2}\right)+i \frac{t^{\frac{5}{2}}}{3 \sqrt{3}}\left(\partial b_{1}\right) b_{1} c_{1} c_{2}+\left(\partial b_{2}\right) c_{2} \\
& -\frac{5 t\left(3+t^{2}\right)}{18}\left(\partial b_{1}\right) b_{1}\left(\partial c_{2}\right) c_{2}-i\left(\frac{\sqrt{2} t(t-1)}{6} \partial \phi_{L, 2}+i \frac{\sqrt{2} t(1+t)}{6} \partial \phi_{M, 2}\right)\left(\partial b_{1}\right) c_{2} \\
& -\frac{i t\left(5 t^{2}-21\right)}{12 \sqrt{3 t}}\left(\partial b_{1}\right)\left(\partial c_{2}\right)+\frac{i t}{2 \sqrt{3 t}}\left(\partial^{2} b_{1}\right) c_{2}+\frac{(1+t)(t-1)}{2 \sqrt{2 t}}\left(\partial^{2} \phi_{L, 1}\right)+\frac{(1+t)(t-3)}{2 \sqrt{6 t}}\left(\partial^{2} \phi_{L, 2}\right) \\
& +\left(\frac{i t(1-t)}{2 \sqrt{3 t}} \partial \phi_{L, 1}{ }^{2}-\frac{i t(3+t)}{6 \sqrt{3 t}} \partial \phi_{L, 2}{ }^{2}+\frac{i t(1+t)}{2 \sqrt{3 t}} \partial \phi_{M, 1}{ }^{2}+\frac{2 t^{2}}{3 \sqrt{3 t}} \partial \phi_{L, 2} \partial \phi_{M, 2}+\frac{i t(t-3)}{6 \sqrt{3 t}} \partial \phi_{M, 2}{ }^{2}\right. \\
& \left.+i \frac{(1+t)(t-1)}{\sqrt{6}}\left(\partial^{2} \phi_{L, 1}\right)+i \frac{t(1+t)}{3 \sqrt{2}}\left(\partial^{2} \phi_{L, 2}\right)-\frac{(1+t)(t-1)}{\sqrt{6}}\left(\partial^{2} \phi_{M, 1}\right)-\frac{t(t-1)}{3 \sqrt{2}}\left(\partial^{2} \phi_{M, 2}\right)\right) b_{1} c_{2} \\
& \left.+i \frac{(1+t)(t-1)}{2 \sqrt{2 t}}\left(\partial^{2} \phi_{M, 1}\right)+i \frac{(3+t)(t-1)}{2 \sqrt{6 t}}\left(\partial^{2} \phi_{M, 2}\right)\right] V_{1,2 ; 1,1}^{L} V_{1,2 ; 1,1}^{M}
\end{aligned}
$$

$$
\begin{aligned}
& \gamma_{2}^{0} \equiv \mathcal{O}_{2,1 ; 1,1}^{(F)}= \\
& {\left[\frac{3+t}{12} \partial \phi_{L, 2}^{2}-\frac{(t-1)}{4} \partial \phi_{L, 1}^{2}-\frac{t}{\sqrt{3}} \partial \phi_{L, 1} \partial \phi_{L, 2}-i \frac{t}{2} \partial \phi_{L, 1} \partial \phi_{M, 1}+\frac{t}{\sqrt{3}} \partial \phi_{M, 1} \partial \phi_{M, 2}\right.} \\
& -i \frac{t}{2 \sqrt{3}} \partial \phi_{L, 2} \partial \phi_{M, 1}+\frac{1+t}{4} \partial \phi_{M, 1}^{2}-i \frac{t}{2 \sqrt{3}} \partial \phi_{L, 1} \partial \phi_{M, 2}-i \frac{t}{6} \partial \phi_{L, 2} \partial \phi_{M, 2} \\
& -\frac{(t-3)}{12} \partial \phi_{M, 2}^{2}+\frac{2 t^{2}}{3} b_{1} b_{2} c_{1} c_{2}-\frac{5 i t^{3}}{3 \sqrt{3 t}} b_{1} b_{2}\left(\partial c_{2}\right) c_{2}+\frac{7 i t}{2 \sqrt{3 t}} b_{1}\left(\partial^{2} c_{2}\right) \\
& +\left(\frac{(t-1) \sqrt{2 t}}{4} \partial \phi_{L, 1}+\frac{(t-1)}{4} \sqrt{\frac{2 t}{3}} \partial \phi_{L, 2}+\frac{(1+t) i \sqrt{2 t}}{4} \partial \phi_{M, 1}+\frac{(1+t) i}{4} \sqrt{\frac{2 t}{3}} \partial \phi_{M, 2}\right) b_{1} c_{1} \\
& +\left(\frac{\left(5 t^{2}+10 t-3\right)}{4 \sqrt{6}} \partial \phi_{M, 1}+\frac{\left(5 t^{2}+10 t-3\right)}{12 \sqrt{2}} \partial \phi_{M, 2}-\frac{i\left(5 t^{2}-10 t-3\right)}{4 \sqrt{6}} \partial \phi_{L, 1}-\frac{i\left(5 t^{2}-10 t-3\right)}{12 \sqrt{2}} \partial \phi_{L, 2}\right) b_{1} \partial c_{2} \\
& +\left(t \sqrt{\frac{t}{2}} \partial \phi_{L, 1}+\frac{t^{2}}{2} \sqrt{\frac{2}{3 t}} \partial \phi_{L, 2}+i t \sqrt{\frac{t}{2}} \partial \phi_{M, 1}+i \frac{t^{2}}{2} \sqrt{\frac{2}{3 t}} \partial \phi_{M, 2}\right) b_{2} c_{2}+\frac{9+5 t^{2}}{6} b_{2}\left(\partial c_{2}\right) \\
& +i \frac{t^{3}}{3 \sqrt{3}}\left(\partial b_{1}\right) b_{1} c_{1} c_{2}+\frac{t\left(5 t^{2}-33\right)}{18}\left(\partial b_{1}\right) b_{1}\left(\partial c_{2}\right) c_{2}+\frac{\left(3+t^{2}\right)}{6}\left(\partial b_{1}\right) c_{1}+i \frac{t^{2}}{\sqrt{3 t}} b_{2} c_{1} \\
& -\left(i \frac{t(t-1)}{4} \sqrt{\frac{2}{3}} \partial \phi_{L, 1}-i \frac{\sqrt{2} t(t-1)}{12} \partial \phi_{L, 2}-\frac{t(1+t)}{4} \sqrt{\frac{2}{3}} \partial \phi_{M, 1}-\frac{\sqrt{2} t(1+t)}{12} \partial \phi_{M, 2}\right)\left(\partial b_{1}\right) c_{2}-
\end{aligned}
$$




$$
\begin{aligned}
& -i \frac{t\left(5 t^{2}-21\right)}{12 \sqrt{3 t}}\left(\partial b_{1}\right)\left(\partial c_{2}\right)+i \frac{t}{2 \sqrt{3 t}}\left(\partial^{2} b_{1}\right) c_{2}+\frac{(t-1)}{2 \sqrt{2 t}}\left(\partial^{2} \phi_{L, 1}\right)+\left(\partial b_{2}\right) c_{2} \\
& -\frac{\left(3+3 t+2 t^{2}\right)}{2 \sqrt{6 t}}\left(\partial^{2} \phi_{L, 2}\right)-i \frac{(1+t)}{2 \sqrt{2 t}}\left(\partial^{2} \phi_{M, 1}\right)-i \frac{3-3 t+2 t^{2}}{2 \sqrt{6 t}}\left(\partial^{2} \phi_{M, 2}\right)+b_{1} c_{2}\left(\frac{t^{\frac{3}{2}}}{6} \partial \phi_{L, 2} \partial \phi_{M, 1}\right. \\
& -i \frac{t(t-1)}{4 \sqrt{3 t}} \partial \phi_{L, 1}{ }^{2}+i \frac{\sqrt{t}(3+t)}{6} \partial \phi_{L, 1} \partial \phi_{L, 2}-i \frac{t(3+5 t)}{12 \sqrt{3 t}} \partial \phi_{L, 2}{ }^{2}-\frac{i \sqrt{t}(t-3)}{6} \partial \phi_{M, 1} \partial \phi_{M, 2} \\
& +\frac{t^{2}}{2 \sqrt{3 t}} \partial \phi_{L, 1} \partial \phi_{M, 1}+\frac{i t(1+t)}{4 \sqrt{3 t}} \partial \phi_{M, 1}^{2}+\frac{t^{\frac{3}{2}}}{6} \partial \phi_{L, 1} \partial \phi_{M, 2}+\frac{t^{2}}{6 \sqrt{3 t}} \partial \phi_{L, 2} \partial \phi_{M, 2} \\
& +i \frac{t(5 t-3)}{12 \sqrt{3 t}} \partial \phi_{M, 2}{ }^{2}+i \frac{(t-1)}{2 \sqrt{6}}\left(\partial^{2} \phi_{L, 1}\right)+i \frac{3+9 t+4 t^{2}}{6 \sqrt{2}}\left(\partial^{2} \phi_{L, 2}\right)+\frac{1+t}{2 \sqrt{6}}\left(\partial^{2} \phi_{M, 1}\right) \\
& \left.\left.-\frac{3-9 t+4 t^{2}}{6 \sqrt{2}}\left(\partial^{2} \phi_{M, 2}\right)\right)\right] V_{2,1 ; 1,1}^{L} V_{2,1 ; 1,1}^{M}
\end{aligned}
$$

\section{References}

[1] D.J. Gross and A.A. Migdal, Phys. Rev. Lett. $\underline{64}$ (1990) 717; M. Douglas and S. Shenker, Nucl. Phys. B235 (1990) 635; E. Brezin and V. Kazakov, Phys. Lett. $\underline{236 B}(1990) 144$.

[2] E. Witten, Nucl. Phys. B340 (1990) 281.

[3] M. Kontsevich, "Intersection Theory on the Moduli Space of Curves and the Matrix Airy Function," Max Plank Institute preprint MPI/91-77

[4] K. Li, Nucl. Phys. B354 (1991) 711.

[5] E. Witten, Comm. Math. Phys. 117 (1988) 353; Comm. Math. Phys. 118 (1988) 411; Nucl. Phys. $\underline{B 340}$ (1990) 281; T. Eguchi and S. Yang, Mod. Phys. Let. $\underline{A 4}$ (1990) 1693.

[6] R. Dijkgraaf, E. Verlinde and H. Verlinde, "Notes on Topological String Theory and 2-D Quantum Gravity," Lectures given at Spring School on Strings and Quantum Gravity, Trieste, Italy, Apr 24 - May 2, 1990 and at Cargese Workshop on Random Surfaces, Quantum Gravity and Strings, Cargese, France, May 28 June 1, 1990.

[7] B. Gato-Rivera and A.M. Semikhatov, Phys. Lett. $\underline{B 293}$ (1992) 72. 
[8] Y. Kazama and H. Suzuki, Phys. Lett. $\underline{216 B}$ (1989) 112; Nucl. Phys. B321 (1989) 232.

[9] E. Witten, Nucl. Phys. B373 (1992) 187.

[10] D. Nemeschansky and N.P. Warner, Nucl. Phys. B380 (1992) 241.

[11] M. Spiegelglas and S. Yankielowicz, $G / G$ topological field theories by cosetting $G(k)$, preprint TECHNION-PH-90-34.

[12] E. Witten, Nucl. Phys. B371 (1992)191.

[13] O. Aharony, O. Ganor, J. Sonnenschein and S. Yankielowicz, preprint TAUP1961-92; O. Aharony, J. Sonnenschein and S. Yankielowicz, Phys. Lett. $\underline{B 289}$ (1992) 309

[14] H. Ooguri and C. Vafa, Mod. Phys. Let. $\underline{A 5}$ (1990) 1389; Nucl. Phys. B361 (1991) 469.

[15] A.B. Zamolodchikov, Theor. Math. Phys. 65 (1985) 1205; V.A. Fateev and A.B. Zamolodchikov, Nucl. Phys. $\underline{B 280}$ (1987) 644; F. Bais, P. Bouwknegt, K. Schoutens and M. Surridge, Nucl. Phys. B304 (1988) 348; V.A. Fateev and S.L. Luk'yanov, Int. J. Mod. Phys. $\underline{A 3}$ (1988) 507.

[16] A. Bilal and J. Gervais, Nucl. Phys. B326 (1989) 222.

[17] S. Das, A. Dhar and S. Kalyana Rama, preprint TIFR/TH/91-20.

[18] H. Lu, C.N. Pope, S. Schrans and K. Xu, preprint CTP-TAMU-5/92, KUL-TF92/1, KUL-TF-92/1; H. Lu, C.N. Pope, S. Schrans and X.J. Wang, preprint CTP-TAMU-15/92; C.N. Pope, preprint CTP-TAMU-30/92.

[19] P. Mansfield and B. Spence, Nucl. Phys. B362 (1991) 294.

[20] D. Friedan, E. Martinec and S. Shenker, Nucl. Phys. B271 (1986) 93.

[21] C. Vafa and S. Mukhi, private communication.

[22] K. Fujikawa and H. Suzuki, Nucl. Phys. B361 (1991) 539; H. Yoshii, "Hidden Higher Supersymmetries in Topological Conformal Field Theories," Tokyo University preprint TIT/HEP-165, December 1990

[23] A. Schwimmer and N. Seiberg, Phys. Lett. $\underline{184 B}$ (1987) 91.

[24] L. Alvarez-Gaume and D.Z. Freedman, Comm. Math. Phys. $\underline{80}$ (1981) 443.

[25] M. Bershadsky, W. Lerche, D. Nemeschansky and N.P. Warner, Phys. Lett. $\underline{B 292}$ (1992) 35 . 
[26] E. Bergshoeff, A. Sevrin and X. Shen, A Derivation of the BRST operator for noncritical $W$ strings, preprint UG-8-92.

[27] D. Nemeschansky and S. Yankielowicz, N=2 W-algebras, Kazama-Suzuki models and Drinfeld-Sokolov reduction, preprint USC-91-005A.

[28] L.J. Romans, Nucl. Phys. B369 (1992) 403.

[29] K. Ito, Phys. Lett. $\underline{B 259}$ (1991) 73; Nucl. Phys. B370 (1992) 123.

[30] W. Lerche, D. Nemeschansky and N.P. Warner, unpublished.

[31] E. Verlinde and H. Verlinde, Nucl. Phys. B348 (1991) 457.

[32] G. Mussardo, G. Sotkov, M. Stanishkov, Int. J. Mod. Phys. A4 (1989) 1135; N. Ohta and H. Suzuki, Nucl. Phys. B332 (1990) 146.

[33] M. Bershadsky and H. Ooguri, Comm. Math. Phys. 126 (1989) 49; M. Bershadsky and H. Ooguri, Phys. Lett. $\underline{229 B}$ (1989) 374.

[34] K. Pilch, private communication.

[35] L. Rozansky, private communicaton

[36] W. Lerche, C. Vafa and N.P. Warner, Nucl. Phys. B324 (1989) 427.

[37] B. Lian and G. Zuckerman, Phys. Lett. $\underline{254 B}$ (1991) 417, Phys. Lett. $\underline{266 B}$ (1991)21, Comm. Math. Phys. 145 (1992) 561.

[38] P. Bouwknegt, J. McCarthy and K. Pilch, Comm. Math. Phys. 145 (1992) 541; Fock space resolutions of the Virasoro highest weight modules with $c \leq 1$, preprint CERN-TH.6196/91; BRST analysis of physical states for 2d (super) gravity coupled to (super) conformal matter, preprint CERN-TH.6279/91.

[39] A. Polyakov, Mod. Phys. Let. $\underline{A 6}$ (1991) 635; I. Klebanov and A. Polyakov, Mod. Phys. Let. $\underline{A 6}$ (1991) 3273; S. Mukherji, S. Mukhi and A. Sen, Phys. Lett. $\underline{266 B}$ (1991) 337; H. Kanno and M. Sarmadi, preprint IC/92/150; K. Itoh and N. Ohta, Nucl. Phys. B377 (1992) 113; N. Chair, V. Dobrev and H. Kanno, Phys. Lett. $\underline{283 B}$ (1992) 194; S. Govindarajan, T. Jayamaran, V. John and P. Majumdar, Mod. Phys. Let. $\underline{A 7}$ (1992) 1063; S. Govindarajan, T. Jayamaran and V. John, Chiral rings and physical states in $c<1$ string theory, preprint IMSc-92/30.

[40] J. Distler and P. Nelson, Comm. Math. Phys. 138 (1991) 273.

[41] D. Kutasov, E. Martinec and N. Seiberg, Phys. Lett. B276 (1992) 437.

[42] N. Seiberg, Progr. Theor. Phys. Suppl. 102 (1990) 319. 
[43] V. Dotsenko, Mod. Phys. Let. $\underline{A 6}$ (1991) 3601; Y. Kitazawa, Phys. Lett. $\underline{B 265}$ (1991) 262.

[44] S. Govindarajan, T. Jayamaran, V. John and P. Majumdar, Mod. Phys. Let.

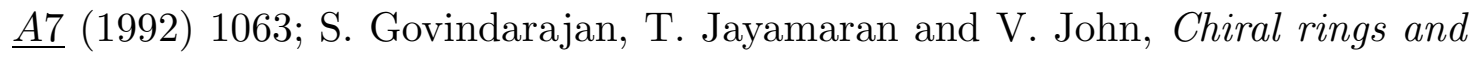
physical states in $c<1$ string theory, preprint IMSc-92/30; Genus zero correlation functions in $c<1$ string theory, preprint IMSC-92-35

[45] J. Distler, Nucl. Phys. B342 (1990) 523.

[46] P. Bouwknegt, J. McCarthy and K. Pilch, On physical states in 2d (topological) gravity, preprint CERN-TH.6645/92.

[47] C.N. Pope, E. Sezgin , K.S. Stelle and X.J. Wang, Discrete states in the $W_{3}$ string, preprint CTP-TAMU-64-92.

[48] D. Gepner, A comment on the chiral algebras of quotient superconformal field theories, preprint PUPT-1130; S. Hosono and A. Tsuchiya, Comm. Math. Phys. $\underline{136}$ (1991) 451.

[49] K. Li, Phys. Lett. B251 (1990) 54, Nucl. Phys. B346 (1990) 329; S. Hosono, Algebraic definition of topological $W$-gravity, preprint UT-588-TOKYO.

[50] W. Lerche, Phys. Lett. $\underline{252 B}$ (1990) 349.

[51] T. Eguchi, S. Hosono and S.K. Yang, Comm. Math. Phys. 140 (1991) 159; T. Eguchi, T. Kawai, S. Mizoguchi and S.K. Yang, Character formulas for coset $N=2$ superconformal theories, preprint KEK-TH-303.

[52] P. Fendley, W. Lerche, S. Mathur and N.P. Warner, Nucl. Phys. B348 (1991) 66.

[53] P. Fendley, S. Mathur C. Vafa and N.P. Warner, Nucl. Phys. B348 (1991) 66.

[54] W. Lerche and N.P. Warner, Nucl. Phys. B358 (1991) 571.

[55] A. Lossev, Descendants constructed from matter field and K. Saito higher residue pairing in Landau-Ginzburg theories coupled to topological gravity, preprint TPIMINN-92-40-T.

[56] L. Dixon, M. Peskin and J. Lykken, Nucl. Phys. $\underline{B 325}$ (1989) 329.

[57] I. Bakas and E. Kiritsis, Beyond the large $N$ limit: Nonlinear $W_{\infty}$ as symmetry of the $S L(2, R) / U(1)$ coset model, preprint UCB-PTH-91-44.

[58] E. Witten, Phys. Rev. D44 (1991) 314.

[59] K. Thielemans, Int. J. Mod. Phys. C Vol. 2, No. 3, 787 (1991). 
[60] P. Bouwknegt, J. McCarthy and K. Pilch, Lett. Math. Phys. 23 (1991) 193.

[61] G. Watts, Nucl. Phys. B326 (1989) 648.

[62] J. Distler and T. Kawai, Nucl. Phys. B321 (1989) 509. 